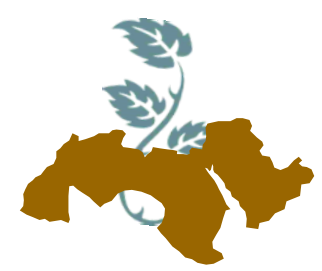

Arab Univ.

J. Agric. Sci., Ain Shams Univ., Cairo, 26(2), 459 - 473, 2018

\title{
EFFECT OF FOLIAR APPLICATION OF ANTIOXIDANTS ON VEGETATIVE GROWTH AND LEAF MINERAL CONTENT OF CHINESE TANGERINE YOUNG TREES BUDDED ON SOME CITRUS ROOTSTOCKS GROWN UNDER SALINE CONDITIONS
}

\author{
Zaky $^{1}$, I.F.; Bedour Abu Leila ${ }^{1}$; N. Abdel Hamid ${ }^{2}$ and H. El-Wakeel ${ }^{2}$ \\ 1- Water Relations Dept., Agriculture and Biological Research Division, National Research \\ Center, Giza, Egypt \\ 2- Horticulture Dept. Fac. of Agric. Ain Shams Univ., Cairo, Egypt
}

Keywords: Citrus, Rootstock, Ascorbic acid, Salicylic acid and Saline

\section{ABSTRACT}

Filed experiment was conducted at El Alamin Wady El Natroun high way (Beer Hooker Area) in a private orchard in a sandy soil under drip irrigation system with saline water. in 2015 and 2016 seasons. Chinese tangerine (Citrus tangerine) transplant were budded on three rootstocks namely sour orange (Citrus aurantum, mion), volkamer lemon (Citrus volkameriana, Tem) and X639 hybrid between (Cleopatra mandarin $\times$ Ponicurus trifoliata) grown under saline conditions to study the effect of foliar application of antioxidants namely ascorbic and salicylic acid on vegetative growth and leaf mineral contents. Results showed that, Volkamer lemon rootstock surpassed sour orange and $x 639$ rootstocks under saline conditions, the highest incremental percentage young tree height, stem diameter, number of leaves per shoot and leaf area were registered by Chinese tangarine scion budded on Volkamer lemon rootstock. Among selected antioxidants ascorbic acid at the concentrations of $800 \mathrm{ppm}$ recorded the highest significant values for vegetative growth parameters and surpassed salicylic acid. Also Chinese mandarin scion budded on volkamer lemon accumulated the highest concentrations of nitrogen $(N)$, phosphorus $(\mathrm{P})$, magnesium $(\mathrm{Mg})$, and chloride $\left(\mathrm{Cl}^{-}\right)$and the lowest concentration of sodium $\left(\mathrm{Na}^{+}\right)$. Chinese mandarin scion budded on sour orange rootstock accumulated the highest concentrations of potassium $(\mathrm{K})$ while Chinese tangerine scion budded on x639 accumulated the highest concentrations of sodium ( $\mathrm{Na})$.

\section{INTRODUCTION}

Citrus grown in many areas of the world. It's a subtropical crop that's not tolerant to freezing temperature or salinity. According to the United Nations of food and agriculture (FAO, 2016) there were 140 citrus producing countries, producing $115,650,545$ tons around $70 \%$ of the world's total citrus production is grown in northern hemisphere, in particular countries around the Mediterranean and United Nations. Citrus even when irrigated water is of good quality, the use of fertilizers and other agro-chemicals raises the likelihood of salt building up in the soil causing salinity stress (Syvertsen et al 1995). Relative to many crop plants, citrus trees have been classified as a salt sensitive crop (Story and Walker, 1999), because saline irrigation water reduced citrus tree growth and fruit yield relatively more than in many other crops (Grieve et al 2007). All citrus varieties must have budded on selected rootstocks and no signal rootstock is suitable for all sites or for all varieties. Rootstock can have a large effect on many aspects of production including yield, fruit quality, tree size, tolerance to salts and scion compatibility. The general tolerance and fruit quality effects of various citrus rootstocks. Thus, it is quite imperative to identify alternative such as rootstocks that can be tolerant to salinity levels, where rootstock vary in their relative resistance to salinity in Egypt, increasing the cultivated area by reclaiming desert are the challenges of near future. Ascorbic acid is 
one of non-enzymatic molecule which plays a substantial role in counteracting oxidative stress caused by stresses. It is a small, water soluble antioxidant molecule which act as a primary substrate in the cyclic pathway for enzymatic detoxification, it acts directly to neutralize superoxide radicals, signal oxygen or superoxide as a secondary antioxidant during reduction cycling of the oxidized for $\alpha$ tocopherol which another lipophilic antioxidant molecule (Noctor and Foyer, 1998). Ascorbic acid has been associated with several types of biological activities in plants such as enzyme CoFactor and as a donor/acceptor in electron transport in plasma membrane or in chloroplast. Much evidence has suggested that ascorbic acid effects biosynthesis, level and signaling of many phytohormones including ethylene, gibberellic acid acid and abscisic acid (Barth et al 2006), all of which are related to oxidative stress resistance Conklin, (2001), Hussein and Alva (2014) Çavuşoğlu and Bilir (2015), El-Sayed and Abdel-Rahman et al (2015), Alhasnawi et al (2015) and Kostopoulou et al (2015).Salicylic acid (SA) or orthohydroxy benzoic acid is distributed in whole plant kingdom. Salicylic acid is considered to be protect plant hormone (Raskin, 1992) because of its divers regulating roles in plant metabolism Popova, et al (1997). Salicylic acid is an endogenous plant growth regulator of phenolic nature that possesses an aromatic ring with hydroxyl group or its functional derivate. The effect of exogenous salicylic acid on growth and bio productivity and may be plays a key role in regulating their growth and productivity. The main object of the present work is to study the effect of salinity on growth, some water relations and chemical constitutes of Chinese mandarin (Chinese tangerine) budded on three citrus rootstocks to test the effect of foliar application with two antioxidants (Salicylic acid and ascorbic acid) on improving their tolerance ability.

\section{MATERIALS AND METHODS}

In this was carried out during a two successive seasons of (2014/2015) and (2015/2016), to evaluate the response of foliar application of some antioxidants on Citrus rootstocks for Chinese tangerine young trees grown under irrigation with saline water. Chinese tangerine (Citrus Tangerine), were budded on three citrus rootstocks namely sour orange (Citrus aurantum, mion), Volkamer lemon (Citrus volkameriana, Tem) and X639 hybrid between (Cleopatra mandarin $\times$ Ponicurus trifoliata). The experiment was conducted at El Alamin -
Wady EI Natroun high way (Beer Hooker Area) in a private orchid in a sandy soil under drip irrigation system with saline water (1700 ppm).

\section{Experimental design}

The experiment of Chinese tangerine included 24 treatments which were three rootstocks (sour orange, volkamer lemon and X639) arranged in split split plot design with a replicate allocated randomly in main plots, the two antioxidants in the sub main plot and the four concentrations $(0,400,600$, $800 \mathrm{ppm}$ ), were distributed randomly in the sub submain plots. Thus the design of the experiment was split split plot with three replicates. The transplants were planted in $28 / 5 / 2014$ at $5 \times 5$ meters apart. The young Chinese tangerine transplants received citrus fertilization program included organic matter fertilizer at $20 \mathrm{~m}^{3} / \mathrm{fed} /$ year and calcium superphosphate $\left(15.5 \% \mathrm{P}_{2} \mathrm{O}_{5}\right)$ in February of each experiment year. Potassium sulfate $\left(48 \% \mathrm{~K}_{2} \mathrm{O}\right)$ at $200 \mathrm{~kg} / \mathrm{fed} / \mathrm{year}$ in March and September according to the recommendations of Ministry of Agriculture and Land Reclamation (MALR,) of Egypt.

\section{Antioxidant Treatments}

The selected antioxidants materials namely ascorbic and salicylic acid were used in the experiments as Foliar spraying two times in both the two seasons (2015 and 2016). The first foliar application at $15^{\text {th }}$ of June and the second foliar application was 15 days later in the first time. The foliar application was conducted at the early morning using back spryer (25 Liter). The volume of the spraying solution was maintained just to cover completely the seedling until drop The selected antioxidant materials were used as Follows:

\subsubsection{Tap water (control)}

\subsubsection{Salicylic acid (SA)}

It is a poorly soluble in water, thus it was soluble in ethyl alcohol and then in distilled water at four concentrations $(0,400,600$ and 800 ppm).

\subsubsection{Ascorbic Acid (ASA)}

It was diluted in distilled water and used at four concentrations $(0,400,600$ and 800 ppm). 

grown under saline conditions

\section{Recorded Data}

\section{Growth Parameters}

At the first week of September the following data were measured:

\section{Young tree height $(\mathrm{cm})$}

The transplant height was measured prior to treatments application $\left(\mathrm{L}^{1}\right)$, then at the end of the growing season $\left(1^{\text {st }}\right.$ week of September) $\left(L^{2}\right)$ and the difference was recorded as an increase in transplant height for each treatment :

\section{$\%$ Increment in transplant height =}

$$
\frac{L 2-L 1}{L 1} \times 100
$$

Stem diameter $(\mathbf{m m})$ : The diameter of the trunk was measured prior to treatments (dia') then at the end of the growing season $\left(1^{\text {st }}\right.$ week of September) $\left(\mathrm{dia}^{2}\right)$ and the difference was recorded as incremental percentage in diameter for each treatment.

$$
\frac{D 2-D 1}{D 1} \times 100
$$

\section{Number of leaves per young tree}

At $1^{\text {st }}$ week of September leaves of each branch were counted, the percentage of increment of leaves was calculated the mean number of the leaves per tree was recorded.

\section{Leaf area $\left(\mathrm{cm}^{2}\right)$}

Was calculated in random samples were taken from the most recently fully mature leaves from the fourth to the sixth leaves from the top of the selected shoots (three leaves per shoot $x$ five shoots) using the equation:

Leaf area $=2 / 3$ length $x$ width., according to Singh and Snyder (1984).

\section{Chemical Analysis}

Samples of twenty mature leaves were selected at random from outer middle of the spring cycle tagged shoots $\left(1^{\text {st }}\right.$ week of September), then they cleaned with piece of cloth to remove dust after that washed twice with tap water, rinsed three times with distilled water and ground in staleness steel mill to avoid contamination with any minerals. Samples dried to a constant weight at $70^{\circ} \mathrm{C}$ in an electric oven and then $0.2 \mathrm{~g}$ of the ash was digested in a mixture of $5: 1$ perchloric acid and sulphoric acid (Jackson, 1973).

1. Total nitrogen was determined by Micro-Kjeldahl method according to (Jackson, 1973).

2. Total phosphorus was determined calorimetrically by ascorbic acid reduction method according to (Murphy and Riley, 1962), as modified by (Watanabe and Olsen, 1965).

3. Total potassium was determined by using flame photometer according to Brown and Lilleland, (1946).

4. Calcium, magnesium, sodium and chloride were determined by titration against versant solution (Chapman and Pratt, 1961).

\section{Statistical analysis}

The obtained data in 2015 and 2016 seasons were subjected to analysis of variance. Means was differentiated using multiply Rang test at the 0.05 level (Duncan, 1955).

\section{RESULTS AND DISCUSSION}

Effect of foliar application of both salicylic and ascorbic acids and their interactions on vegetative growth of Chinese tangerine budded on volkamer lemon, sour orange and X639 rootstocks grown under saline conditions.

\section{Vegetative growth parameters}

\subsection{Effect of rootstocks}

It is evident from presented data in (Table 1, 2, 3 and 4) that Chinese tangerine budded on volkamer lemon rootstock under saline conditions surpassed the other rootstocks and gave the significantly highest values for young tree \% increment of height, \% increment of stem diameter, \% increment of leaf number and leaf area $\left(\mathrm{cm}^{2}\right) \mathrm{com}$ pared with other rootstocks namely sour orange which ranked in between and X639 which recorded the lowest values.

Thus, it mean that, there is a great variation in ability of citrus rootstocks to tolerant salinity .In this respect Muhamed and AkberAnjun (2008) recorded that reduction in fresh weight were more in troyer citrange as compared with Cleopatra mandarin under saline conditions, this revealed that in our study volkamer lemon rootstock is most tolerant than sour orange and $\mathrm{X} 639$, there may be that the growth and yield of citrus crop inhibited due to 
the high ratio of $\mathrm{Na} / \mathrm{Ca}$ and $\mathrm{Na} / \mathrm{K}$ under saline conditions. These results hold true with the finding of Zikri and Parsonss (1992) on citrus. In these respect, Storey, (1995) recorded that, the tolerance of different species of citrus can be determined by their ability to exclude the potentially toxic $\mathrm{Na}^{+}$and $\mathrm{Cl}^{-}$ions.

\subsection{Effect of antioxidants and their concen- trations}

In the present study foliar application of ascorbic acid surpassed salicylic acid in increasing vegetative growth parameters i.e young tress increment $\%$ of height, increment $\%$ of stem diameter, increment $\%$ of leaf number and leaf area $\left(\mathrm{cm}^{2}\right)$ compared with the control salinized rootstocks. However, the increase in growth parameters were associated with the increase in antioxidants concentrations up to $800 \mathrm{ppm}$ dose, it could be concluded that foliar application of ascorbic acid reduced the damage effect of salinity on vegetative growth procedure, associated the rate growth and reversed the effect of salinity more than salicylic acid.

1.3. Interaction effect of rootstock, antioxidants and their concentration

The interaction between studied rootstocks, antioxidants and their different concentrations, the data revealed that, volkamer lemon rootstock interacted with ascorbic acid at the concentration of $800 \mathrm{ppm}$ in both seasons and gave the highest significant values for young tress increment \% of height, increment $\%$ of stem diameter, increment $\%$ of leaf number per shoot and leaf area $\left(\mathrm{cm}^{2}\right)$. In our results ascorbic acid surpassed salicylic acid. These results are in agreement with Muhamed Akber (2008) who reported that ascorbic acid application not only mitigate the inhibitory effect of salt stress but also induced stimulatory effect on all of the studied growth parameters of soccum spp, hybrid cv., HSF-24 grown under salt stress. Moreover ascorbic acid in regard as one of the most effective growth regulator against abiotic stress. Ascorbic acid not only acts as antioxidants but also cellular level of ascorbic acid are correlated with the activation of complex biological defense mechanism. Further more experimental studies on different plants have shown that exogenous application of ascorbic acid may reduce salt induced adverse effect and results insignificant increments on growth and yield Salma, 2009 and Athar et al 2009).

\section{Leaf Mineral Contents}

Regarding the ion uptake by stressed rootstocks all studied were achieved to determine whether the differential plants response to salinity can correlated with the modification of plant mineral composition and the obtained results indicated that under stress conditions more cations and less anions were accumulated by young tress of Chinese tangerine leaves. Our results in (Table 5) revealed that nitrogen $(\mathrm{N})$ concentration in Chinese tangerine budded on volkamer lemon rootstock was the highest while sour orange rootstock recorded the lowest value however, x639 ranked in between.

Table (6) show that phosphorus $(P)$ concentration was the highest for Chinese tangerine budded on volkamer lemon rootstock while $x 639$ tended to be the lowest one. The highest potassium (K) concentration (Table 7) was registered for $x 639$ rootstock followed in descending order by volkamer lemon rootstock. In this study lowest values registered by sour orange rootstock. (Table 8 ) show that calcium content $(\mathrm{Ca})$ for x639 rootstock recorded the highest values. Whereas sour orange recorded the lowest values compared with the other rootstocks, in theses respect volkamer lemon rootstock ranked in between. The highest magnesium (Mg) concentration (Table 9) was registered for Chinese tangerine budded on Volkamer lemon rootstock, while sour orange rootstock recorded the lowest value of magnesium $(\mathrm{Mg})$ concentration. As for sodium (Na) (Table 10) concentration X639 significantly values of $\mathrm{Na}$ the lowest mean values recorded for Chinese tangerine budded on volkamer lemon rootstock.

Chloride $\left(\mathrm{Cl}^{-}\right)$concentration recorded the lowest mean values for Chinese tangerine budded on sour orange rootstock, while Chinese tangerine budded on volkamer lemon rootstock registered the highest mean value despite of the lowest value of sodium $\mathrm{Na}$.

Thus, it means that there are great differences between the studied rootstocks to accumulate nutrients under saline conditions. The stimulatory effect of low salinity level on some minerals $N, P$, and $\mathrm{K}$ of many plants were reported by Heikal et al (1980). The observed increase in sodium $\mathrm{Na}$ under saline condition was reported by Storey (1995) who observed that the accumulation of $\mathrm{Na}$ by plant under saline conditions may be attributed to the damage of protoplasm of plant cells and as a result, the selective salt absorption in replace by passive absorption which causes abnormal 
Effect of foliar application of antioxidants on vegetative growth and leaf mineral content of chinese tangerine young trees budded on some citrus rootstocks grown under saline conditions

Table 1. Effect of antioxidant foliar application and rootstocks on tree height increment percentage of Chinese tangerine young trees under saline conditions

\begin{tabular}{|c|c|c|c|c|c|c|c|c|c|c|c|}
\hline \multicolumn{2}{|c|}{ Treatments } & \multicolumn{5}{|c|}{ First season, 2015} & \multicolumn{5}{|c|}{ Second season; 2016} \\
\hline Rootstock & Antioxidant & 0 & 400ppm & 600 ppm & 800ppm & MeanAxB & 0 & 400ppm & $600 p p m$ & $800 p p m$ & MeanAxB \\
\hline \multirow{2}{*}{$\begin{array}{c}\text { Sour } \\
\text { orange }\end{array}$} & SA & $10.14 \mathrm{k}$ & $11.56 \mathrm{ij}$ & $15.13 d-f$ & $15.80 \mathrm{~cd}$ & 13.16DE & $14.21 j$ & 14.96hi & $16.97 f$ & $21.23 d$ & 16.84D \\
\hline & ASC & $10.20 \mathrm{k}$ & $12.13 \mathrm{i}$ & $16.25 \mathrm{bc}$ & 16.89ab & $13.86 \mathrm{C}$ & $12.87 \mathrm{k}$ & 15.23hi & $17.43 \mathrm{f}$ & $22.65 c$ & 17.05D \\
\hline \multirow{2}{*}{$\begin{array}{c}\text { Volkamer } \\
\text { Lemon }\end{array}$} & SA & $13.26 \mathrm{~g}$ & $14.86 \mathrm{~d}-f$ & $15.47 c-e$ & $16.23 \mathrm{bc}$ & 14.95B & $15.56 \mathrm{gh}$ & $17.09 f$ & $21.22 d$ & $24.17 b$ & 19.51B \\
\hline & ASC & 14.70 ef & $14.27 f$ & $15.20 d-f$ & $17.56 \mathrm{a}$ & $15.43 \mathrm{~A}$ & $16.14 \mathrm{~g}$ & $16.99 f$ & $22.51 \mathrm{c}$ & $26.18 a$ & $20.45 \mathrm{~A}$ \\
\hline \multirow{2}{*}{ X639 } & SA & $10.86 \mathrm{jk}$ & 12.32hi & $13.14 \mathrm{gh}$ & $14.86 d-f$ & $12.79 \mathrm{E}$ & $14.18 \mathrm{j}$ & 15.16hi & $16.89 f$ & $19.07 e$ & 16.32E \\
\hline & ASC & $11.47 \mathrm{ij}$ & $11.91 \mathrm{i}$ & $14.34 \mathrm{f}$ & $15.74 \mathrm{~cd}$ & 13.36D & $14.55 i j$ & $16.85 f$ & $18.88 \mathrm{e}$ & $21.17 d$ & $17.86 \mathrm{C}$ \\
\hline \multirow{3}{*}{\multicolumn{2}{|c|}{ Mean $(A \times C)$}} & $10.17 \mathrm{G}$ & $11.84 \mathrm{E}$ & 15.69B & $16.35 \mathrm{~A}$ & & 13.541 & $15.09 \mathrm{G}$ & $17.20 \mathrm{E}$ & 21.94B & \\
\hline & & $13.98 \mathrm{CD}$ & $14.57 \mathrm{C}$ & 15.33B & $16.90 \mathrm{~A}$ & & $15.85 \mathrm{~F}$ & 17.04E & 21.87B & $25.17 \mathrm{~A}$ & \\
\hline & & $11.16 \mathrm{~F}$ & $12.11 \mathrm{E}$ & 13.74D & $15.30 \mathrm{~B}$ & & $14.36 \mathrm{H}$ & $16.00 \mathrm{~F}$ & $17.89 \mathrm{D}$ & $20.12 \mathrm{C}$ & \\
\hline \multirow{2}{*}{\multicolumn{2}{|c|}{ Mean $(B \times C)$}} & $11.42 \mathrm{~F}$ & 12.91D & $14.58 \mathrm{C}$ & 15.63B & & $14.65 \mathrm{G}$ & $15.73 \mathrm{~F}$ & $18.36 \mathrm{D}$ & $21.49 \mathrm{~B}$ & \\
\hline & & $12.12 \mathrm{E}$ & 12.77D & $15.26 \mathrm{~B}$ & $16.73 \mathrm{~A}$ & & $14.52 \mathrm{G}$ & 16.36E & $19.61 \mathrm{C}$ & $23.33 \mathrm{~A}$ & \\
\hline \multicolumn{2}{|c|}{ Mean (C) } & 11.77D & $12.84 \mathrm{C}$ & 14.92B & $16.18 \mathrm{~A}$ & & $14.59 \mathrm{D}$ & $16.05 \mathrm{C}$ & 18.98B & $22.41 \mathrm{~A}$ & \\
\hline \multicolumn{2}{|c|}{ Mean (B) } & \multicolumn{2}{|c|}{ 13.63B } & \multicolumn{2}{|c|}{$14.32 \mathrm{~A}$} & & & $56 \mathrm{~B}$ & \multicolumn{2}{|c|}{$18.45 \mathrm{~A}$} & \\
\hline \multicolumn{2}{|c|}{ Mean A } & 13.51B & \multicolumn{2}{|c|}{$15.19 \mathrm{~A}$} & $13.08 \mathrm{C}$ & & $16.94 \mathrm{~B}$ & \multicolumn{2}{|c|}{$19.98 \mathrm{~A}$} & $17.09 \mathrm{~B}$ & \\
\hline
\end{tabular}

Mean in each row, colum or interaction having the same letter (s) are not significantly different at $5 \%$ level.

$A=$ Rootstocks $\quad B=$ Antioxidants $\quad C=$ Concentrations $\quad A \times B=$ Rootstocks $\times$ Antioxidant

$B \times C=$ Antioxidant $\times$ Concentrations $\quad A \times B \times C=$ Rootstocks $\times$ Antioxidant $\times$ Concentrations.

$\mathrm{SA}=$ Salicylic acid $\mathrm{ASC}=$ Ascorbic acid

Table 2. Effect of antioxidant foliar application and rootstocks on tree stem diameter increment percentage of Chinese tangerine young trees under saline conditions

\begin{tabular}{|c|c|c|c|c|c|c|c|c|c|c|c|}
\hline \multicolumn{2}{|c|}{ Treatments } & \multicolumn{5}{|c|}{ First season, 2015} & \multicolumn{5}{|c|}{ Second season; 2016} \\
\hline Rootstock & Antioxidant & 0 & 400ppm & $600 p p m$ & $800 p p m$ & MeanAxB & 0 & 400ppm & $600 \mathrm{ppm}$ & $800 p p m$ & MeanAxB \\
\hline \multirow{2}{*}{ Sour orange } & SA & $12.81 \mathrm{gh}$ & $14.91 d-f$ & 15.20de & $16.85 b$ & 14.94B & $12.61 \mathrm{k}$ & $15.21 \mathrm{~g}$ & 16.42de & $16.86 \mathrm{~cd}$ & $15.28 \mathrm{D}$ \\
\hline & ASC & $11.97 i$ & $14.78 \mathrm{ef}$ & $16.11 b c$ & $16.14 b c$ & $14.75 \mathrm{~B}$ & $12.43 \mathrm{k}$ & $15.35 \mathrm{fg}$ & $18.59 \mathrm{~b}$ & $17.53 \mathrm{c}$ & $15.97 \mathrm{C}$ \\
\hline \multirow{2}{*}{$\begin{array}{l}\text { Volkamer } \\
\text { Lemon }\end{array}$} & SA & $13.25 \mathrm{~g}$ & 15.08de & $15.59 \mathrm{~cd}$ & $17.94 a$ & $15.47 \mathrm{~A}$ & $13.94 j$ & 16.24de & $17.42 \mathrm{c}$ & $18.34 b$ & $16.49 \mathrm{~B}$ \\
\hline & ASC & $12.91 \mathrm{~g}$ & 14.43ef & $17.78 \mathrm{a}$ & $17.95 a$ & $15.77 \mathrm{~A}$ & $14.03 \mathrm{j}$ & $15.23 \mathrm{~g}$ & $18.73 b$ & $19.47 a$ & $16.87 \mathrm{~A}$ \\
\hline \multirow{2}{*}{ X639 } & SA & $12.03 i$ & $13.50 \mathrm{~g}$ & $14.22 f$ & $14.61 \mathrm{ef}$ & $13.59 \mathrm{C}$ & $12.24 \mathrm{k}$ & $14.11 \mathrm{ij}$ & $14.79 \mathrm{~g}-\mathrm{i}$ & 15.97ef & $14.28 \mathrm{E}$ \\
\hline & ASC & 12.16hi & $13.15 \mathrm{~g}$ & $13.17 \mathrm{~g}$ & $14.41 \mathrm{ef}$ & $13.22 \mathrm{D}$ & $12.89 \mathrm{k}$ & $14.36 h-j$ & $15.21 \mathrm{~g}$ & $15.03 \mathrm{gh}$ & 14.37E \\
\hline \multirow{3}{*}{\multicolumn{2}{|c|}{ Mean $(A \times C)$}} & $12.39 \mathrm{G}$ & 14.85D & $15.65 \mathrm{C}$ & $16.50 \mathrm{~B}$ & & $12.52 \mathrm{G}$ & $15.28 \mathrm{DE}$ & $17.51 \mathrm{C}$ & $17.19 \mathrm{C}$ & \\
\hline & & $13.08 \mathrm{~F}$ & 14.76D & $16.69 \mathrm{~B}$ & $17.94 \mathrm{~A}$ & & $13.99 \mathrm{~F}$ & 15.73D & 18.08B & $18.91 \mathrm{~A}$ & \\
\hline & & $12.09 \mathrm{G}$ & 13.32EF & $13.69 \mathrm{E}$ & $14.51 \mathrm{D}$ & & $12.57 \mathrm{G}$ & $14.23 \mathrm{~F}$ & $15.00 \mathrm{E}$ & 15.50DE & \\
\hline \multirow{2}{*}{\multicolumn{2}{|c|}{ Mean $(B \times C)$}} & $12.70 \mathrm{E}$ & $14.50 \mathrm{D}$ & $15.00 \mathrm{C}$ & $16.47 \mathrm{~A}$ & & $12.93 \mathrm{E}$ & 15.19D & $16.21 \mathrm{C}$ & $17.06 \mathrm{~B}$ & \\
\hline & & $12.35 \mathrm{E}$ & 14.12D & $15.69 \mathrm{~B}$ & $16.17 \mathrm{~A}$ & & $13.12 \mathrm{E}$ & 14.98D & $17.51 \mathrm{~A}$ & $17.34 \mathrm{AB}$ & \\
\hline \multicolumn{2}{|c|}{ Mean ( C ) } & $12.52 \mathrm{D}$ & $14.31 \mathrm{C}$ & 15.34B & $16.32 \mathrm{~A}$ & & $13.02 \mathrm{D}$ & $15.08 \mathrm{C}$ & $16.86 \mathrm{~B}$ & $17.20 \mathrm{~A}$ & \\
\hline \multicolumn{2}{|c|}{ Mean (B) } & \multicolumn{2}{|c|}{$14.67 \mathrm{~A}$} & \multicolumn{2}{|c|}{$14.58 \mathrm{~A}$} & & & $35 \mathrm{~B}$ & \multicolumn{2}{|c|}{$15.74 \mathrm{~A}$} & \\
\hline \multicolumn{2}{|c|}{ Mean A } & $14.85 \mathrm{~B}$ & \multicolumn{2}{|c|}{$15.62 \mathrm{~A}$} & $13.40 \mathrm{C}$ & & $15.63 \mathrm{~B}$ & \multicolumn{2}{|c|}{$16.68 \mathrm{~A}$} & $14.33 C$ & \\
\hline
\end{tabular}

Mean in each row, Colum or interaction having the same letter (s) are not significantly different at $5 \%$ level.

$A=$ Rootstocks $\quad B=$ Antioxidants $\quad C=$ Concentrations $A \times B=$ Rootstocks $\times$ Antioxidant

$\mathrm{B} \times \mathrm{C}=$ Antioxidant $\times$ Concentrations $\mathrm{A} \times \mathrm{B} \times \mathrm{C}=$ Rootstocks $\times$ Antioxidant $\times$ Concentrations.

$\mathrm{SA}=$ Salicylic acid $\mathrm{ASC}=$ Ascorbic acid.

Arab Univ. J. Agric. Sci., 26(2), 2018 
Table 3. Effect of antioxidant foliar application and rootstocks on $\%$ of increment of leaves number of Chinese tangerine young trees grown under saline conditions

\begin{tabular}{|c|c|c|c|c|c|c|c|c|c|c|c|}
\hline \multicolumn{2}{|c|}{ Treatments } & \multicolumn{5}{|c|}{ First season, 2015} & \multicolumn{5}{|c|}{ Second season; 2016} \\
\hline Rootstock & Antioxidant & 0 & 400ppm & $600 \mathrm{ppm}$ & $800 p p m$ & Mean AxB & 0 & 400ppm & $600 \mathrm{ppm}$ & $800 p p m$ & MeanAxB \\
\hline \multirow{2}{*}{$\begin{array}{c}\text { Sour } \\
\text { orange }\end{array}$} & SA & 13.210 & $15.61 \mathrm{~h}-\mathrm{k}$ & $15.49 i-k$ & $21.88 d$ & & $15.22 r$ & $15.61 \mathrm{qr}$ & $18.98 \mathrm{gh}$ & $20.17 e$ & 17.49E \\
\hline & ASC & $14.34 \mathrm{mn}$ & $14.82 \mathrm{Im}$ & $22.81 \mathrm{c}$ & $23.57 b$ & 18.88B & 16.41op & $16.50 n-p$ & $19.22 \mathrm{fg}$ & $23.59 a b$ & $18.93 \mathrm{C}$ \\
\hline \multirow{2}{*}{$\begin{array}{c}\text { Volkamer } \\
\text { Lemon }\end{array}$} & SA & $16.09 g-j$ & $18.14 \mathrm{e}$ & $24.59 a$ & $24.94 a$ & $20.94 \mathrm{~A}$ & $17.26 \mathrm{Im}$ & 19.64ef & $21.07 d$ & $22.50 \mathrm{c}$ & $20.12 B$ \\
\hline & ASC & $16.40 \mathrm{fg}$ & $16.93 f$ & $24.54 a$ & $24.82 a$ & $20.67 \mathrm{~A}$ & $17.05 \mathrm{mn}$ & $18.31 \mathrm{ij}$ & $23.29 \mathrm{~b}$ & $23.88 \mathrm{a}$ & $20.63 \mathrm{~A}$ \\
\hline \multirow{2}{*}{$\mathrm{X} 639$} & SA & $14.04 n$ & $15.63 \mathrm{~h}-\mathrm{k}$ & $16.08 \mathrm{~g}-\mathrm{j}$ & $16.17 \mathrm{~g}-\mathrm{i}$ & $15.48 \mathrm{D}$ & $15.93 p q$ & $16.88 \mathrm{~m}-\mathrm{o}$ & $17.86 \mathrm{jk}$ & $19.24 \mathrm{fg}$ & 17.48E \\
\hline & ASC & $15.12 \mathrm{kl}$ & $15.84 \mathrm{~g}-\mathrm{j}$ & 16.19gh & $15.45 \mathrm{jk}$ & 15.65D & $15.92 p q$ & $17.71 \mathrm{kl}$ & 18.50hi & $19.34 \mathrm{fg}$ & 17.87D \\
\hline \multirow{3}{*}{\multicolumn{2}{|c|}{ Mean $(A \times C)$}} & $13.77 \mathrm{I}$ & $15.21 \mathrm{G}$ & $19.15 \mathrm{C}$ & $22.73 \mathrm{~B}$ & & $15.82 \mathrm{~F}$ & $16.06 \mathrm{~F}$ & $19.10 \mathrm{C}$ & 21.88B & \\
\hline & & $16.25 \mathrm{E}$ & $17.53 \mathrm{D}$ & $24.57 \mathrm{~A}$ & $24.88 \mathrm{~A}$ & & 17.16E & $18.97 \mathrm{C}$ & $22.18 \mathrm{~B}$ & $23.19 \mathrm{~A}$ & \\
\hline & & $14.58 \mathrm{H}$ & $15.74 \mathrm{~F}$ & 16.14EF & 15.81EF & & $15.93 \mathrm{~F}$ & $17.30 \mathrm{E}$ & $18.18 \mathrm{D}$ & $19.29 \mathrm{C}$ & \\
\hline \multirow{2}{*}{\multicolumn{2}{|c|}{ Mean $(B \times C)$}} & $14.45 \mathrm{~F}$ & $16.46 \mathrm{C}$ & $18.72 \mathrm{~B}$ & $21.00 \mathrm{~A}$ & & $16.14 \mathrm{~F}$ & 17.37D & $19.30 \mathrm{C}$ & 20.64B & \\
\hline & & $15.29 \mathrm{E}$ & 15.86D & $21.18 \mathrm{~A}$ & $21.28 \mathrm{~A}$ & & 16.46E & $17.51 \mathrm{D}$ & 20.34B & 22.27A & \\
\hline \multicolumn{2}{|c|}{ Mean (C) } & 14.87D & $16.16 \mathrm{C}$ & 19.95B & $21.14 \mathrm{~A}$ & & 16.30D & $17.44 \mathrm{C}$ & 19.82B & $21.45 \mathrm{~A}$ & \\
\hline \multicolumn{2}{|c|}{ Mean (B) } & \multicolumn{2}{|c|}{$17.66 \mathrm{~B}$} & \multicolumn{2}{|c|}{$18.40 \mathrm{~A}$} & & \multicolumn{2}{|c|}{$18.36 \mathrm{~B}$} & \multicolumn{2}{|c|}{$19.14 \mathrm{~A}$} & \\
\hline \multicolumn{2}{|c|}{ Mean A } & $17.72 \mathrm{~B}$ & \multicolumn{2}{|c|}{$20.81 \mathrm{~A}$} & $15.57 \mathrm{C}$ & & 18.21B & \multicolumn{2}{|c|}{$20.37 \mathrm{~A}$} & $17.67 \mathrm{C}$ & \\
\hline
\end{tabular}

Mean in each row, colum or interaction having the same letter (s) are not significantly different at $5 \%$ level.

$A=$ Rootstocks $\quad B=$ Antioxidants $\quad C=$ Concentrations $\quad A \times B=$ Rootstocks $\times$ Antioxidant

$B \times C=$ Antioxidant $\times$ Concentrations $\quad A \times B \times C=$ Rootstocks $\times$ Antioxidant $\times$ Concentrations.

SA= Salicylic acid ASC $=$ Ascorbic acid.

Table 4. Effect of foliar application of antioxidants and rootstock on leaf area $\left(\mathrm{cm}^{2}\right)$ of Chinese tangerine young trees grown under saline conditions

\begin{tabular}{|c|c|c|c|c|c|c|c|c|c|c|c|}
\hline \multicolumn{2}{|c|}{ Treatments } & \multicolumn{5}{|c|}{ First season, 2015} & \multicolumn{5}{|c|}{ Second season; 2016} \\
\hline Rootstock & Antioxidant & 0 & 400ppm & $600 \mathrm{ppm}$ & 800 ppm & MeanAxB & 0 & 400ppm & $600 \mathrm{ppm}$ & 800 ppm & MeanAxB \\
\hline \multirow{2}{*}{$\begin{array}{c}\text { Sour } \\
\text { orange }\end{array}$} & SA & $10.33 \mathrm{fg}$ & 11.00ef & $13.33 b$ & $13.33 b$ & $12.00 \mathrm{~B}$ & $12.33 \mathrm{ef}$ & $13.33 \mathrm{~cd}$ & $14.00 \mathrm{bc}$ & $14.33 \mathrm{~b}$ & $13.50 \mathrm{~B}$ \\
\hline & ASC & $11.00 \mathrm{ef}$ & $10.33 f g$ & $13.33 b$ & $13.00 \mathrm{bc}$ & 11.92B & $12.33 \mathrm{ef}$ & $13.00 \mathrm{de}$ & $13.33 \mathrm{~cd}$ & $14.67 \mathrm{~b}$ & 13.33B \\
\hline \multirow{2}{*}{$\begin{array}{l}\text { Volkamer } \\
\text { Lemon }\end{array}$} & SA & $12.00 \mathrm{~d}$ & $12.00 d$ & $13.00 \mathrm{bc}$ & $14.67 a$ & $12.92 \mathrm{~A}$ & 13.00de & 13.00de & $14.33 \mathrm{~b}$ & $16.00 \mathrm{a}$ & $14.08 \mathrm{~A}$ \\
\hline & ASC & $11.00 \mathrm{ef}$ & $12.00 \mathrm{~d}$ & $13.00 \mathrm{bc}$ & $14.67 a$ & $12.67 \mathrm{~A}$ & $12.66 \mathrm{fg}$ & $13.33 \mathrm{~cd}$ & $14.67 \mathrm{~b}$ & $16.00 \mathrm{a}$ & $14.00 \mathrm{~A}$ \\
\hline \multirow{2}{*}{ X639 } & SA & $10.00 \mathrm{~g}$ & $10.67 \mathrm{fg}$ & $12.33 c d$ & 11.67de & $11.17 \mathrm{C}$ & $11.67 \mathrm{fg}$ & 12.33ef & $13.33 \mathrm{~cd}$ & $13.33 c d$ & $12.67 \mathrm{C}$ \\
\hline & ASC & $11.00 \mathrm{ef}$ & $12.00 d$ & $12.33 \mathrm{~cd}$ & $12.33 \mathrm{~cd}$ & $11.92 \mathrm{~B}$ & $11.33 \mathrm{~g}$ & $12.00 \mathrm{fg}$ & $13.33 \mathrm{~cd}$ & $14.00 \mathrm{bc}$ & $12.67 \mathrm{C}$ \\
\hline \multicolumn{2}{|c|}{ Mean $(A \times C)$} & $10.67 \mathrm{~F}$ & $10.67 \mathrm{~F}$ & 13.33B & 13.17B & & 12.33D & $13.17 \mathrm{C}$ & $13.67 \mathrm{C}$ & $14.50 \mathrm{~B}$ & \\
\hline \multicolumn{2}{|c|}{ Mean $(A \times C)$} & 11.50DE & $12.00 \mathrm{CD}$ & $13.00 \mathrm{~B}$ & $14.67 \mathrm{~A}$ & & $12.50 \mathrm{D}$ & $13.17 \mathrm{C}$ & $14.50 \mathrm{~B}$ & $16.00 \mathrm{~A}$ & \\
\hline \multicolumn{2}{|c|}{ Mean $(A \times C)$} & $10.50 \mathrm{~F}$ & 11.33E & $12.33 \mathrm{C}$ & $12.00 \mathrm{CD}$ & & $11.50 \mathrm{E}$ & 12.17D & $13.33 \mathrm{C}$ & $13.67 \mathrm{C}$ & \\
\hline \multirow{2}{*}{\multicolumn{2}{|c|}{ Mean $(B \times C)$}} & $10.78 \mathrm{C}$ & $11.22 \mathrm{BC}$ & $12.89 \mathrm{~A}$ & $13.22 \mathrm{~A}$ & & 12.33D & $12.89 \mathrm{C}$ & $13.89 \mathrm{~B}$ & $14.56 \mathrm{~A}$ & \\
\hline & & $11.00 \mathrm{BC}$ & $11.44 \mathrm{~B}$ & $12.89 \mathrm{~A}$ & $13.33 \mathrm{~A}$ & & $11.89 \mathrm{E}$ & $12.78 \mathrm{C}$ & $13.78 \mathrm{~B}$ & $14.89 \mathrm{~A}$ & \\
\hline \multicolumn{2}{|c|}{ Mean (C) } & 10.89D & $11.33 \mathrm{C}$ & $12.89 \mathrm{~B}$ & $13.28 \mathrm{~A}$ & & 12.11D & $12.83 \mathrm{C}$ & 13.83B & $14.72 \mathrm{~A}$ & \\
\hline \multicolumn{2}{|c|}{ Mean (B) } & \multicolumn{2}{|c|}{$12.03 \mathrm{~A}$} & \multicolumn{2}{|c|}{$12.17 \mathrm{~A}$} & & \multicolumn{2}{|c|}{$13.42 \mathrm{~A}$} & \multicolumn{2}{|c|}{$13.33 \mathrm{~A}$} & \\
\hline \multicolumn{2}{|c|}{ Mean A } & $11.96 \mathrm{~B}$ & \multicolumn{2}{|c|}{$12.79 \mathrm{~A}$} & $11.54 \mathrm{C}$ & & $13.42 \mathrm{~B}$ & \multicolumn{2}{|c|}{$14.04 \mathrm{~A}$} & $12.67 \mathrm{C}$ & \\
\hline
\end{tabular}

Mean in each row, Colum or interaction having the same letter (s) are not significantly different at $5 \%$ level..

$\mathrm{A}=$ Rootstocks $\quad \mathrm{B}=$ Antioxidants $\quad \mathrm{C}=$ Concentrations $\mathrm{A} \times \mathrm{B}=$ Rootstocks $\times$ Antioxidant

$\mathrm{B} \times \mathrm{C}=$ Antioxidant $\times$ Concentrations $\mathrm{A} \times \mathrm{B} \times \mathrm{C}=$ Rootstocks $\times$ Antioxidant $\times$ Concentrations.

SA $=$ Salicylic acid ASC $=$ Ascorbic acid. 

content of chinese tangerine young trees budded on some citrus rootstocks grown under saline conditions

Table 5. Effect of foliar application of antioxidants and rootstocks on leaf nitrogen percentage (\%) of Chinese tangerine young trees grown under saline conditions

\begin{tabular}{|c|c|c|c|c|c|c|c|c|c|c|c|}
\hline \multicolumn{2}{|c|}{ Treatments } & \multicolumn{5}{|c|}{ First season, 2015} & \multicolumn{5}{|c|}{ Second season; 2016} \\
\hline Rootstock & Antioxidant & 0 & 400ppm & 600ppm & 800ppm & MeanAxB & 0 & 400ppm & $600 \mathrm{ppm}$ & $800 p p m$ & MeanAxB \\
\hline Sour & SA & $2.13 \mathrm{~h}$ & $2.14 \mathrm{~h}$ & 2.22gh & $2.24 \mathrm{gh}$ & $2.18 \mathrm{D}$ & $2.14 \mathrm{j}$ & $2.24 \mathrm{ij}$ & $2.27 \mathrm{ij}$ & $2.28 \mathrm{~h}-\mathrm{j}$ & $2.23 \mathrm{D}$ \\
\hline orange & ASC & $2.30 \mathrm{fg}$ & $2.40 \mathrm{ef}$ & $2.74 \mathrm{a}$ & $2.66 \mathrm{a}-\mathrm{c}$ & $2.53 \mathrm{~B}$ & $2.32 \mathrm{~g}-\mathrm{j}$ & $2.46 \mathrm{~d}-\mathrm{h}$ & $1.87 \mathrm{k}$ & $2.69 a-c$ & $2.33 \mathrm{C}$ \\
\hline Volkamer & SA & $2.30 f g$ & $2.64 a-c$ & $2.66 a-c$ & $2.72 a$ & $2.58 \mathrm{AB}$ & $2.35 f-i$ & $2.68 \mathrm{a}-\mathrm{c}$ & $2.70 a-c$ & $2.82 \mathrm{a}$ & $2.64 A B$ \\
\hline Lemon & ASC & $2.52 \mathrm{c}-\mathrm{e}$ & $2.52 \mathrm{c}-\mathrm{e}$ & $2.69 a b$ & $2.77 \mathrm{a}$ & $2.63 \mathrm{~A}$ & $2.52 \mathrm{c}-\mathrm{f}$ & $2.64 a-d$ & 2.79ab & $2.77 a b$ & $2.68 \mathrm{~A}$ \\
\hline \multirow{2}{*}{$\mathbf{X 6 3 9}$} & SA & $2.25 \mathrm{gh}$ & $2.44 d-f$ & $2.53 c-e$ & $2.56 b-d$ & $2.45 \mathrm{C}$ & $2.42 \mathrm{e}-\mathrm{i}$ & $2.55 c-e$ & 2.60b-e & $2.67 a-c$ & $2.56 \mathrm{~B}$ \\
\hline & ASC & $2.42 d-f$ & $2.52 \mathrm{c}-\mathrm{e}$ & $2.53 c-e$ & $2.67 a-c$ & $2.54 \mathrm{~B}$ & $2.47 \mathrm{~d}-\mathrm{g}$ & $2.64 a-d$ & $2.68 \mathrm{a}-\mathrm{c}$ & $2.69 a-c$ & $2.62 A B$ \\
\hline \multirow{3}{*}{\multicolumn{2}{|c|}{ Mean $(A \times C)$}} & 2.221 & $2.27 \mathrm{HI}$ & 2.48D-F & 2.45EF & & $2.23 G$ & $2.35 \mathrm{~F}$ & $2.07 \mathrm{H}$ & 2.49DE & \\
\hline & & $2.41 \mathrm{FG}$ & $2.58 \mathrm{~B}-\mathrm{D}$ & $2.68 \mathrm{AB}$ & $2.75 \mathrm{~A}$ & & 2.44EF & $2.66 \mathrm{BC}$ & $2.75 \mathrm{AB}$ & $2.80 \mathrm{~A}$ & \\
\hline & & 2.34GH & $2.48 \mathrm{D}-\mathrm{F}$ & 2.53C-E & $2.62 \mathrm{BC}$ & & $2.45 \mathrm{EF}$ & $2.60 \mathrm{CD}$ & 2.64BC & $2.68 \mathrm{~A}-\mathrm{C}$ & \\
\hline \multirow{2}{*}{\multicolumn{2}{|c|}{ Mean $(B \times C)$}} & $2.23 \mathrm{C}$ & $2.41 \mathrm{~B}$ & $2.47 \mathrm{~B}$ & $2.51 \mathrm{~B}$ & & $2.30 \mathrm{D}$ & $2.49 \mathrm{BC}$ & $2.52 \mathrm{BC}$ & $2.59 \mathrm{~B}$ & \\
\hline & & $2.41 \mathrm{~B}$ & $2.48 \mathrm{~B}$ & $2.65 \mathrm{~A}$ & $2.70 \mathrm{~A}$ & & $2.44 \mathrm{C}$ & $2.58 \mathrm{~B}-\mathrm{D}$ & $2.45 \mathrm{C}$ & $2.72 \mathrm{~A}$ & \\
\hline \multicolumn{2}{|c|}{ Mean ( C ) } & $2.32 \mathrm{C}$ & 2.44B & $2.56 \mathrm{~A}$ & $2.60 \mathrm{~A}$ & & $2.37 \mathrm{C}$ & $2.54 \mathrm{~B}$ & $2.48 \mathrm{~B}$ & $2.65 \mathrm{~A}$ & \\
\hline \multicolumn{2}{|c|}{ Mean (B) } & \multicolumn{2}{|c|}{$2.40 \mathrm{~B}$} & \multicolumn{2}{|c|}{$2.56 \mathrm{~A}$} & & \multicolumn{2}{|c|}{$2.48 \mathrm{~B}$} & \multicolumn{2}{|c|}{$2.55 \mathrm{~A}$} & \\
\hline \multicolumn{2}{|c|}{ Mean A } & $2.35 \mathrm{C}$ & \multicolumn{2}{|c|}{$2.60 \mathrm{~A}$} & $2.49 \mathrm{~B}$ & & $2.28 \mathrm{C}$ & \multicolumn{2}{|c|}{$2.66 \mathrm{~A}$} & $2.59 \mathrm{~B}$ & \\
\hline
\end{tabular}

Mean in each row, colum or interaction having the same letter (s) are not significantly different at $5 \%$ level.
A= Rootstocks
B= Antioxidants
$\mathrm{C}=$ Concentrations $\mathrm{A} \times \mathrm{B}=$ Rootstocks $\times$ Antioxidant
$\mathrm{B} \times \mathrm{C}=$ Antioxidant $\times$ Concentrations $\mathrm{A} \times \mathrm{B} \times \mathrm{C}=$ Rootstocks $\times$ Antioxidant $\times$ Concentrations.

$\mathrm{SA}=$ Salicylic acid $\mathrm{ASC}=$ Ascorbic acid.

$\begin{array}{ll}\text { Element } & \mathbf{N}(\%) \\ \text { Deficient } & <2.2 \\ \text { Low } & 2.2-2.4 \\ \text { Optimum } & 2.5-2.7 \\ \text { High } & 2.8-3.0 \\ \text { Excess } & >3.0\end{array}$


Table 6. Effect of foliar application of antioxidants and rootstocks on leaf phosphorus (\%) of Chinese tangerine young trees grown under saline conditions

\begin{tabular}{|c|c|c|c|c|c|c|c|c|c|c|c|}
\hline \multicolumn{2}{|c|}{ Treatments } & \multicolumn{5}{|c|}{ First season, 2015} & \multicolumn{5}{|c|}{ Second season; 2016} \\
\hline Rootstock & Antioxidant & 0 & $400 p p m$ & $600 \mathrm{ppm}$ & 800ppm & MeanAxB & 0 & 400ppm & $600 \mathrm{ppm}$ & 800ppm & MeanAxB \\
\hline $\begin{array}{c}\text { Sour } \\
\text { orange }\end{array}$ & $\begin{array}{l}\text { SA } \\
\text { ASC }\end{array}$ & $\begin{array}{l}0.090 \mathrm{~h}-\mathrm{j} \\
0.100 \mathrm{~g}-\mathrm{i}\end{array}$ & $\begin{array}{l}0.100 \mathrm{~g}-\mathrm{i} \\
0.100 \mathrm{~g}-\mathrm{i}\end{array}$ & $\begin{array}{l}0.110 f-h \\
0.083 h-j\end{array}$ & $\begin{array}{l}0.110 f-h \\
0.120 e-g \\
\end{array}$ & $\begin{array}{l}0.103 \mathrm{C} \\
0.101 \mathrm{C} \\
\end{array}$ & $\begin{array}{l}0.080 \mathrm{i} \\
0.090 \mathrm{hi} \\
\end{array}$ & $\begin{array}{l}0.110 f g \\
0.110 f g \\
\end{array}$ & $\begin{array}{l}0.120 f \\
0.300 a\end{array}$ & $\begin{array}{l}0.077 i \\
0.110 f g \\
\end{array}$ & $\begin{array}{l}0.097 \mathrm{E} \\
0.153 \mathrm{~A} \\
\end{array}$ \\
\hline $\begin{array}{c}\text { Volkamer } \\
\text { Lemon }\end{array}$ & $\begin{array}{l}\text { SA } \\
\text { ASC }\end{array}$ & $\begin{array}{l}0.100 \mathrm{~g}-\mathrm{i} \\
0.110 f-h\end{array}$ & $\begin{array}{l}0.120 \mathrm{e}-\mathrm{g} \\
0.130 \mathrm{~d}-\mathrm{f}\end{array}$ & $\begin{array}{l}0.140 \mathrm{c}-\mathrm{e} \\
0.150 \mathrm{~cd}\end{array}$ & $\begin{array}{l}0.180 \mathrm{ab} \\
0.190 \mathrm{a}\end{array}$ & $\begin{array}{c}0.135 \mathrm{AB} \\
0.145 \mathrm{~A} \\
\end{array}$ & $\begin{array}{l}0.110 \mathrm{fg} \\
0.100 \mathrm{gh}\end{array}$ & $\begin{array}{l}0.110 f g \\
0.120 f\end{array}$ & $\begin{array}{l}0.140 \mathrm{e} \\
0.160 \mathrm{~cd} \\
\end{array}$ & $\begin{array}{l}0.170 \mathrm{bc} \\
0.180 \mathrm{~b}\end{array}$ & $\begin{array}{c}0.133 \mathrm{BC} \\
0.140 \mathrm{~B}\end{array}$ \\
\hline X639 & $\begin{array}{l}\text { SA } \\
\text { ASC } \\
\end{array}$ & \begin{tabular}{|l|}
$0.090 \mathrm{~h}-\mathrm{j}$ \\
$0.070 \mathrm{j}$
\end{tabular} & $\begin{array}{l}0.110 f-h \\
0.080 i j\end{array}$ & $\begin{array}{l}0.140 c-\mathrm{e} \\
0.140 \mathrm{c}-\mathrm{e}\end{array}$ & $\begin{array}{l}0.160 \mathrm{bc} \\
0.140 \mathrm{c}-\mathrm{e} \\
\end{array}$ & $\begin{array}{l}0.125 \mathrm{~B} \\
0.108 \mathrm{C} \\
\end{array}$ & $\begin{array}{l}0.080 \mathrm{i} \\
0.080 \mathrm{i} \\
\end{array}$ & $\begin{array}{l}0.120 \mathrm{f} \\
0.090 \mathrm{hi} \\
\end{array}$ & $\begin{array}{l}0.150 \mathrm{de} \\
0.160 \mathrm{~cd}\end{array}$ & $\begin{array}{l}0.170 \mathrm{bc} \\
0.160 \mathrm{~cd} \\
\end{array}$ & $\begin{array}{c}0.130 \mathrm{CD} \\
0.123 \mathrm{D} \\
\end{array}$ \\
\hline \multicolumn{2}{|c|}{ Mean $(A \times C)$} & $\begin{array}{c}0.095 \mathrm{FG} \\
0.105 \mathrm{EF} \\
0.080 \mathrm{G} \\
\end{array}$ & $\begin{array}{l}0.100 \mathrm{EF} \\
0.125 \mathrm{CD} \\
0.095 \mathrm{FG} \\
\end{array}$ & $\begin{array}{c}0.097 \mathrm{FG} \\
0.145 \mathrm{~B} \\
0.140 \mathrm{BC} \\
\end{array}$ & $\begin{array}{c}0.115 \mathrm{DE} \\
0.185 \mathrm{~A} \\
0.150 \mathrm{~B} \\
\end{array}$ & & $\begin{array}{l}0.085 \mathrm{GH} \\
0.105 \mathrm{EF} \\
0.080 \mathrm{H} \\
\end{array}$ & \begin{tabular}{|l|}
$0.110 \mathrm{E}$ \\
$0.115 \mathrm{E}$ \\
$0.105 \mathrm{EF}$ \\
\end{tabular} & $\begin{array}{l}0.210 \mathrm{~A} \\
0.150 \mathrm{D} \\
0.155 \mathrm{CD} \\
\end{array}$ & $\begin{array}{c}0.093 \mathrm{FG} \\
0.175 \mathrm{~B} \\
0.165 \mathrm{BC} \\
\end{array}$ & \\
\hline \multicolumn{2}{|c|}{ Mean $(B \times C)$} & $\begin{array}{l}0.093 \mathrm{D} \\
0.093 \mathrm{D} \\
\end{array}$ & $\begin{array}{l}0.110 \mathrm{C} \\
0.103 \mathrm{CD} \\
\end{array}$ & \begin{tabular}{|l|}
$0.130 \mathrm{~B}$ \\
$0.124 \mathrm{~B}$ \\
\end{tabular} & $\begin{array}{l}0.150 \mathrm{~A} \\
0.150 \mathrm{~A} \\
\end{array}$ & & $\begin{array}{l}0.090 \mathrm{E} \\
0.090 \mathrm{E} \\
\end{array}$ & $\begin{array}{l}0.113 \mathrm{D} \\
0.107 \mathrm{D} \\
\end{array}$ & $\begin{array}{l}0.137 \mathrm{C} \\
0.207 \mathrm{~A} \\
\end{array}$ & $\begin{array}{l}0.139 \mathrm{C} \\
0.150 \mathrm{~B} \\
\end{array}$ & \\
\hline \multicolumn{2}{|c|}{ Mean ( C ) } & $0.093 \mathrm{D}$ & $0.107 \mathrm{C}$ & $0.127 \mathrm{~B}$ & $0.150 \mathrm{~A}$ & & $0.090 \mathrm{D}$ & $0.110 \mathrm{C}$ & $0.172 \mathrm{~A}$ & $0.144 \mathrm{~B}$ & \\
\hline \multicolumn{2}{|c|}{ Mean (B) } & \multicolumn{2}{|c|}{$0.121 \mathrm{~A}$} & \multicolumn{2}{|c|}{$0.118 \mathrm{~A}$} & & \multicolumn{2}{|c|}{$0.120 \mathrm{~B}$} & \multicolumn{2}{|c|}{$0.138 \mathrm{~A}$} & \\
\hline Mea & in $A$ & $0.102 \mathrm{C}$ & 0.14 & & $0.116 \mathrm{~B}$ & & $0.125 \mathrm{~B}$ & & $36 \mathrm{~A}$ & $0.126 \mathrm{~B}$ & \\
\hline
\end{tabular}

Mean in each row, colum or interaction having the same letter (s) are not significantly different at $5 \%$ level.

$A=$ Rootstocks $\quad B=$ Antioxidants $\quad C=$ Concentrations $\quad A \times B=$ Rootstocks $\times$ Antioxidant

$B \times C=$ Antioxidant $\times$ Concentrations $A \times B \times C=$ Rootstocks $\times$ Antioxidant $\times$ Concentrations.

$\mathrm{SA}=$ Salicylic acid $\mathrm{ASC}=$ Ascorbic acid.

$\begin{array}{ll}\text { Element } & \mathbf{P}(\%) \\ \text { Deficient } & <0.09 \\ \text { Low } & 0.09-0.11 \\ \text { Optimum } & 0.12-0.16 \\ \text { High } & 0.17-0.30 \\ \text { Excess } & >0.30\end{array}$

Obreza et al (1992) 

content of chinese tangerine young trees budded on some citrus rootstocks grown under saline conditions

Table 7. Effect of foliar application of antioxidants and rootstocks on leaf potassium percentage (\%) of Chinese Tangerine young trees grown under saline conditions

\begin{tabular}{|c|c|c|c|c|c|c|c|c|c|c|c|}
\hline \multicolumn{2}{|c|}{ Treatments } & \multicolumn{5}{|c|}{ First season, 2015} & \multicolumn{5}{|c|}{ Second season; 2016} \\
\hline Rootstock & Antioxidant & 0 & 400ppm & 600ppm & 800ppm & MeanAxB & 0 & 400ppm & $600 p p m$ & 800ppm & \\
\hline \multirow{2}{*}{$\begin{array}{l}\text { Sour } \\
\text { orange } \\
\end{array}$} & SA & $0.660 \mathrm{~h}$ & $0.680 \mathrm{~h}$ & $0.720 f-h$ & $0.770 \mathrm{~d}-\mathrm{g}$ & $0.708 \mathrm{C}$ & $0.800 h-j$ & $0.890 \mathrm{~h}$ & $1.120 \mathrm{~g}$ & $1.200 \mathrm{e}-\mathrm{g}$ & 1.003E \\
\hline & ASC & $0.670 \mathrm{~h}$ & $0.720 f-h$ & $0.897 \mathrm{ab}$ & $0.810 c-e$ & $0.774 \mathrm{~B}$ & $0.720 \mathrm{j}$ & 0.880hi & $1.823 a$ & $1.190 \mathrm{e}-\mathrm{g}$ & $1.153 \mathrm{BC}$ \\
\hline \multirow{2}{*}{$\begin{array}{c}\text { Volkamer } \\
\text { Lemon } \\
\end{array}$} & SA & $0.700 \mathrm{gh}$ & $0.820 \mathrm{~b}-\mathrm{e}$ & $0.880 a-c$ & $0.850 \mathrm{~b}-\mathrm{d}$ & $0.813 \mathrm{AB}$ & $0.750 \mathrm{ij}$ & $0.890 \mathrm{~h}$ & $1.240 \mathrm{~d}-\mathrm{g}$ & $1.340 \mathrm{~b}-\mathrm{d}$ & 1.055DE \\
\hline & ASC & $0.740 \mathrm{e}-\mathrm{h}$ & $0.850 \mathrm{~b}-\mathrm{d}$ & $0.880 \mathrm{a}-\mathrm{c}$ & $0.940 \mathrm{a}$ & $0.853 \mathrm{~A}$ & $0.880 \mathrm{hi}$ & $1.150 \mathrm{fg}$ & $1.270 \mathrm{c}-\mathrm{f}$ & $1.390 \mathrm{bc}$ & 1.173AB \\
\hline \multirow{2}{*}{ X639 } & SA & $0.770 \mathrm{~d}-\mathrm{g}$ & $0.820 \mathrm{~b}-\mathrm{e}$ & $0.820 \mathrm{~b}-\mathrm{e}$ & $0.860 \mathrm{a}-\mathrm{c}$ & $0.818 \mathrm{AB}$ & $0.840 h-j$ & $0.880 \mathrm{hi}$ & $1.320 \mathrm{~b}-\mathrm{e}$ & $1.370 \mathrm{~b}-\mathrm{d}$ & $1.102 \mathrm{CD}$ \\
\hline & ASC & $0.740 \mathrm{e}-\mathrm{h}$ & $0.770 \mathrm{~d}-\mathrm{g}$ & $0.840 \mathrm{~b}-\mathrm{d}$ & $0.800 \mathrm{c}-\mathrm{f}$ & $0.788 \mathrm{AB}$ & $0.880 \mathrm{hi}$ & $1.280 \mathrm{~b}-\mathrm{f}$ & $1.340 \mathrm{~b}-\mathrm{d}$ & $1.410 \mathrm{~b}$ & $1.227 \mathrm{~A}$ \\
\hline \multirow{3}{*}{\multicolumn{2}{|c|}{ Mean $(A \times C)$}} & $0.665 \mathrm{~F}$ & $0.700 \mathrm{EF}$ & $0.808 \mathrm{~A}-\mathrm{D}$ & $0.790 \mathrm{~B}-\mathrm{E}$ & & $0.760 \mathrm{G}$ & $0.885 \mathrm{~F}$ & $1.472 \mathrm{~A}$ & 1.195D & \\
\hline & & $0.720 \mathrm{D}-\mathrm{F}$ & $0.835 \mathrm{~A}-\mathrm{C}$ & $0.880 \mathrm{AB}$ & $0.895 \mathrm{~A}$ & & $0.815 \mathrm{FG}$ & $1.020 \mathrm{E}$ & $1.255 \mathrm{CD}$ & $1.365 \mathrm{~B}$ & \\
\hline & & $0.755 \mathrm{C}-\mathrm{F}$ & $0.795 \mathrm{~A}-\mathrm{E}$ & $0.830 \mathrm{~A}-\mathrm{C}$ & $0.830 \mathrm{~A}-\mathrm{C}$ & & $0.860 \mathrm{~F}$ & $1.080 \mathrm{E}$ & $1.330 \mathrm{BC}$ & $1.390 \mathrm{~B}$ & \\
\hline \multirow{2}{*}{\multicolumn{2}{|c|}{ Mean $(B \times C)$}} & $0.710 \mathrm{C}$ & $0.773 \mathrm{BC}$ & $0.807 \mathrm{AB}$ & $0.827 A B$ & & $0.797 \mathrm{~F}$ & $0.887 \mathrm{E}$ & $1.227 \mathrm{C}$ & $1.303 \mathrm{~B}$ & \\
\hline & & $0.717 \mathrm{C}$ & $0.780 \mathrm{BC}$ & $0.872 \mathrm{~A}$ & $0.850 \mathrm{AB}$ & & $0.827 \mathrm{EF}$ & $1.103 \mathrm{D}$ & $1.478 \mathrm{~A}$ & $1.330 \mathrm{~B}$ & \\
\hline \multicolumn{2}{|c|}{ Mean (C) } & $0.713 \mathrm{C}$ & $0.777 \mathrm{~B}$ & $0.839 \mathrm{~A}$ & $0.838 \mathrm{~A}$ & & $0.812 \mathrm{C}$ & $0.995 \mathrm{~B}$ & $1.352 \mathrm{~A}$ & $1.317 \mathrm{~A}$ & \\
\hline \multicolumn{2}{|c|}{ Mean (B) } & \multicolumn{2}{|c|}{$0.779 \mathrm{~A}$} & \multicolumn{2}{|c|}{$0.805 \mathrm{~A}$} & & \multicolumn{2}{|c|}{$1.053 \mathrm{~B}$} & \multicolumn{2}{|c|}{$1.184 \mathrm{~A}$} & \\
\hline \multicolumn{2}{|c|}{ Mean (A) } & $0.741 \mathrm{~B}$ & 0.83 & $33 \mathrm{~A}$ & $0.803 \mathrm{~A}$ & & $1.078 \mathrm{~B}$ & 1.11 & 14B & $1.165 \mathrm{~A}$ & \\
\hline
\end{tabular}

Mean in each row, colum or interaction having the same letter (s) are not significantly different at $5 \%$ level..

$A=$ Rootstocks $\quad B=$ Antioxidants $\quad C=$ Concentrations $\quad A \times B=$ Rootstocks $\times$ Antioxidant

$\mathrm{B} \times \mathrm{C}=$ Antioxidant $\times$ Concentrations $\quad \mathrm{A} \times \mathrm{B} \times \mathrm{C}=$ Rootstocks $\times$ Antioxidant $\times$ Concentrations .

$\mathrm{SA}=$ Salicylic acid $\mathrm{ASC}=$ Ascorbic acid.

$\begin{array}{lll}\text { Element } & \mathrm{K}(\%) & \text { Obreza et al (1992) } \\ \text { Deficient } & <0.7 & \\ \text { Low } & 0.7-1.1 & \\ \text { Optimum } & 1.2-1.7 & \\ \text { High } & 1.8-2.4 & \\ \text { Excess } & >2.4 & \end{array}$


Table 8. Effect of foliar application of antioxidants and rootstocks on leaf calcium percentage a (\%) of Chinese tangerine young trees grown under saline conditions

\begin{tabular}{|c|c|c|c|c|c|c|c|c|c|c|c|}
\hline \multicolumn{2}{|c|}{ Treatments } & \multicolumn{5}{|c|}{ First season, 2015} & \multicolumn{5}{|c|}{ Second season; 2016} \\
\hline Rootstock & Antioxidant & 0 & 400ppm & 600ppm & $800 p p m$ & MeanAxB & 0 & 400ppm & 600ppm & $800 \mathrm{ppm}$ & MeanAxB \\
\hline $\begin{array}{c}\text { Sour } \\
\text { orange }\end{array}$ & $\begin{array}{l}\text { SA } \\
\text { ASC } \\
\end{array}$ & $\begin{array}{r}1.44 \mathrm{i} \\
1.72 \mathrm{~h} \\
\end{array}$ & \begin{tabular}{|c}
$2.10 \mathrm{f}$ \\
$2.88 \mathrm{~b}-\mathrm{d}$ \\
\end{tabular} & $\begin{array}{c}2.84 d \\
3.05 a-d \\
\end{array}$ & $\begin{array}{l}2.92 b-d \\
3.07 a-d \\
\end{array}$ & $\begin{array}{l}2.33 \mathrm{D} \\
2.68 \mathrm{C} \\
\end{array}$ & $\begin{array}{r}2.22 \mathrm{j} \\
1.82 \mathrm{k} \\
\end{array}$ & \begin{tabular}{|l|}
$2.82 \mathrm{e}-\mathrm{g}$ \\
$2.68 \mathrm{f}-\mathrm{h}$ \\
\end{tabular} & \begin{tabular}{|c|}
$2.98 \mathrm{c}-\mathrm{e}$ \\
$2.31 \mathrm{j}$ \\
\end{tabular} & $\begin{array}{l}2.95 \mathrm{~d}-\mathrm{f} \\
3.16 \mathrm{~b}-\mathrm{d} \\
\end{array}$ & $\begin{array}{l}2.74 \mathrm{~B} \\
2.49 \mathrm{C} \\
\end{array}$ \\
\hline $\begin{array}{c}\text { Volkamer } \\
\text { Lemon }\end{array}$ & $\begin{array}{l}\text { SA } \\
\text { ASC } \\
\end{array}$ & $\begin{array}{l}1.76 \mathrm{gh} \\
1.98 \mathrm{gg} \\
\end{array}$ & $\begin{array}{l}2.86 \mathrm{~cd} \\
3.02 \mathrm{a}-\mathrm{d} \\
\end{array}$ & $\begin{array}{l}2.94 b-d \\
3.14 a b \\
\end{array}$ & $\begin{array}{r}3.22 \mathrm{a} \\
3.14 \mathrm{ab} \\
\end{array}$ & $\begin{array}{c}2.70 \mathrm{BC} \\
2.82 \mathrm{~A} \\
\end{array}$ & $\begin{array}{r}1.84 \mathrm{k} \\
2.45 \mathrm{~h}-\mathrm{j} \\
\end{array}$ & $\begin{array}{l}2.57 \mathrm{~g}-\mathrm{i} \\
3.25 \mathrm{a}-\mathrm{d} \\
\end{array}$ & \begin{tabular}{|l|}
$3.17 b-d$ \\
$3.31 a b$ \\
\end{tabular} & $\begin{array}{l}3.31 a b \\
3.34 a b \\
\end{array}$ & $\begin{array}{l}2.72 \mathrm{~B} \\
3.09 \mathrm{~A} \\
\end{array}$ \\
\hline X639 & $\begin{array}{c}\text { SA } \\
\text { ASC }\end{array}$ & $\begin{array}{l}2.14 \mathrm{f} \\
2.44 \mathrm{e} \\
\end{array}$ & $\begin{array}{l}2.99 a-d \\
2.87 b-d\end{array}$ & \begin{tabular}{|l|}
$3.04 a-d$ \\
$3.13 a-c$ \\
\end{tabular} & \begin{tabular}{|l}
$3.06 a-d$ \\
$3.10 a-d$
\end{tabular} & $\begin{array}{c}2.81 \mathrm{AB} \\
2.89 \mathrm{~A} \\
\end{array}$ & $\begin{array}{l}2.25 \mathrm{j} \\
3.21 \mathrm{a}-\mathrm{d}\end{array}$ & $\begin{array}{l}3.27 a-c \\
2.94 d-f\end{array}$ & \begin{tabular}{|l|}
$3.22 a-d$ \\
$3.25 a-d$
\end{tabular} & $\begin{array}{r}3.49 a \\
3.34 a b \\
\end{array}$ & $\begin{array}{l}3.06 \mathrm{~A} \\
3.18 \mathrm{~A} \\
\end{array}$ \\
\hline \multicolumn{2}{|c|}{ Mean $(A \times C)$} & $\begin{array}{l}1.58 \mathrm{~F} \\
1.87 \mathrm{E} \\
2.29 \mathrm{D}\end{array}$ & $\begin{array}{l}2.49 \mathrm{C} \\
2.94 \mathrm{~B} \\
2.93 \mathrm{~B} \\
\end{array}$ & $\begin{array}{c}2.94 \mathrm{~B} \\
3.04 \mathrm{AB} \\
3.09 \mathrm{AB} \\
\end{array}$ & $\begin{array}{c}2.99 \mathrm{~B} \\
3.18 \mathrm{~A} \\
3.08 \mathrm{AB} \\
\end{array}$ & & $\begin{array}{l}2.02 \mathrm{~F} \\
2.15 \mathrm{~F} \\
3.73 \mathrm{DE}\end{array}$ & $\begin{array}{l}2.75 \mathrm{DE} \\
2.91 \mathrm{CD} \\
3.11 \mathrm{BC}\end{array}$ & \begin{tabular}{|c|}
$2.65 \mathrm{E}$ \\
$3.24 \mathrm{AB}$ \\
$3.24 \mathrm{AB}$ \\
\end{tabular} & $\begin{array}{c}3.06 \mathrm{BC} \\
3.33 \mathrm{~A} \\
3.42 \mathrm{~A} \\
\end{array}$ & \\
\hline \multicolumn{2}{|c|}{ Mean $(B \times C)$} & \begin{tabular}{|l|}
$1.78 \mathrm{~F}$ \\
$2.05 \mathrm{E}$ \\
\end{tabular} & $\begin{array}{l}2.65 \mathrm{D} \\
2.92 \mathrm{C} \\
\end{array}$ & $\begin{array}{c}2.94 \mathrm{BC} \\
3.11 \mathrm{~A} \\
\end{array}$ & \begin{tabular}{|c|}
$3.07 \mathrm{AB}$ \\
$3.10 \mathrm{~A}$ \\
\end{tabular} & & $\begin{array}{l}2.10 \mathrm{D} \\
2.49 \mathrm{C} \\
\end{array}$ & $\begin{array}{r}2.89 \mathrm{~B} \\
2.96 \mathrm{~B} \\
\end{array}$ & $\begin{array}{l}3.12 \mathrm{~A} \\
2.96 \mathrm{~B} \\
\end{array}$ & $\begin{array}{l}3.25 \mathrm{~A} \\
3.28 \mathrm{~A} \\
\end{array}$ & \\
\hline \multicolumn{2}{|c|}{ Mean (C) } & $1.91 \mathrm{C}$ & $2.79 \mathrm{~B}$ & $3.02 \mathrm{~A}$ & $3.09 \mathrm{~A}$ & & $2.30 \mathrm{D}$ & $2.92 \mathrm{C}$ & 3.04B & $3.27 \mathrm{~A}$ & \\
\hline \multicolumn{2}{|c|}{ Mean (B) } & \multicolumn{2}{|c|}{$2.61 \mathrm{~B}$} & \multicolumn{2}{|c|}{$2.80 \mathrm{~A}$} & & \multicolumn{2}{|c|}{$2.84 \mathrm{~B}$} & \multicolumn{2}{|c|}{$2.92 \mathrm{~A}$} & \\
\hline Me & & $2.50 \mathrm{C}$ & 2.7 & & $2.85 \mathrm{~A}$ & & $2.62 \mathrm{C}$ & & & $3.12 \mathrm{~A}$ & \\
\hline
\end{tabular}

Mean in each row , Colum or interaction having the same letter (s) are not significantly different at $5 \%$ level..

$A=$ Rootstocks $\quad B=$ Antioxidants $\quad C=$ Concentrations $\quad A \times B=$ Rootstocks $\times$ Antioxidant

$\mathrm{B} \times \mathrm{C}=$ Antioxidant $\times$ Concentrations $\mathrm{A} \times \mathrm{B} \times \mathrm{C}=$ Rootstocks $\times$ Antioxidant $\times$ Concentrations.

$\mathrm{SA}=$ Salicylic acid $\mathrm{ASC}=$ Ascorbic acid.

$\begin{array}{ll}\text { Element } & \text { Ca (\%) } \\ \text { Deficient } & <1.5 \\ \text { Low } & 1.5-2.9 \\ \text { Optimum } & 3.0-4.9\end{array}$

Obreza et al (1992) 

content of chinese tangerine young trees budded on some citrus rootstocks grown under saline conditions

Table 9. Effect of foliar application of antioxidants and rootstocks on leaf magnesium percentage (\%) of Chinese tangerine young trees grown under saline conditions

\begin{tabular}{|c|c|c|c|c|c|c|c|c|c|c|c|}
\hline \multicolumn{2}{|c|}{ Treatments } & \multicolumn{5}{|c|}{ First season 2015} & \multicolumn{5}{|c|}{ Second season 2016} \\
\hline Rootstock & Antioxidant & 0 & 400ppm & 600ppm & 800ppm & MeanAxB & 0 & $400 p p m$ & 600ppm & 800ppm & Mear \\
\hline $\begin{array}{c}\text { Sour } \\
\text { orange }\end{array}$ & $\begin{array}{l}\text { SA } \\
\text { ASC }\end{array}$ & $\begin{array}{c}0.440 \mathrm{f}-\mathrm{i} \\
0.290 \mathrm{j} \\
\end{array}$ & $\begin{array}{l}0.410 \mathrm{hi} \\
0.510 \mathrm{~d}-\mathrm{h} \\
\end{array}$ & $\begin{array}{l}0.407 \mathrm{hi} \\
0.530 \mathrm{~d}-\mathrm{g} \\
\end{array}$ & $\begin{array}{c}0.390 \mathrm{i} \\
0.580 \mathrm{~b}-\mathrm{e} \\
\end{array}$ & $\begin{array}{l}0.412 \mathrm{D} \\
0.478 \mathrm{C} \\
\end{array}$ & $\begin{array}{l}0.430 \mathrm{e} \\
0.270 \mathrm{f} \\
\end{array}$ & $\begin{array}{l}0.440 \mathrm{de} \\
0.520 \mathrm{~b}-\mathrm{d} \\
\end{array}$ & $\begin{array}{l}0.450 \mathrm{de} \\
0.527 \mathrm{~b}-\mathrm{d}\end{array}$ & $\begin{array}{l}0.440 \mathrm{de} \\
0.590 \mathrm{ab} \\
\end{array}$ & $\begin{array}{l}0.440 \mathrm{C} \\
0.477 \mathrm{C} \\
\end{array}$ \\
\hline $\begin{array}{c}\text { Volkamer } \\
\text { Lemon }\end{array}$ & $\begin{array}{r}\text { SA } \\
\text { ASC } \\
\end{array}$ & $\begin{array}{c}0.510 \mathrm{~d}-\mathrm{h} \\
0.470 \mathrm{f}-\mathrm{i} \\
\end{array}$ & $\begin{array}{l}0.630 \mathrm{bc} \\
0.510 \mathrm{~d}-\mathrm{h} \\
\end{array}$ & $\begin{array}{l}0.660 \mathrm{ab} \\
0.540 \mathrm{c}-\mathrm{f} \\
\end{array}$ & $\begin{array}{c}0.640 \mathrm{ab} \\
0.730 \mathrm{a} \\
\end{array}$ & $\begin{array}{l}0.610 \mathrm{~A} \\
0.532 \mathrm{~B} \\
\end{array}$ & $\begin{array}{l}0.520 \mathrm{~b}-\mathrm{d} \\
0.490 \mathrm{c}-\mathrm{e}\end{array}$ & $\begin{array}{c}0.650 \mathrm{a} \\
0.550 \mathrm{bc} \\
\end{array}$ & $\begin{array}{c}0.660 \mathrm{a} \\
0.590 \mathrm{ab} \\
\end{array}$ & $\begin{array}{l}0.650 \mathrm{a} \\
0.550 \mathrm{bc} \\
\end{array}$ & $\begin{array}{l}0.620 \mathrm{~A} \\
0.545 \mathrm{~B} \\
\end{array}$ \\
\hline X639 & $\begin{array}{l}\text { SA } \\
\text { ASC }\end{array}$ & $\begin{array}{l}0.460 \mathrm{f}-\mathrm{i} \\
0.430 \mathrm{~g}-\mathrm{i}\end{array}$ & $\begin{array}{l}0.480 f-\mathrm{i} \\
0.490 \mathrm{e}-\mathrm{i}\end{array}$ & $\begin{array}{l}0.520 \mathrm{~d}-\mathrm{g} \\
0.520 \mathrm{~d}-\mathrm{g} \\
\end{array}$ & $\begin{array}{l}0.600 \mathrm{~b}-\mathrm{d} \\
0.500 \mathrm{~d}-\mathrm{h}\end{array}$ & $\begin{array}{l}0.515 C \\
0.485 C \\
\end{array}$ & $\begin{array}{l}0.480 \mathrm{c}-\mathrm{e} \\
0.450 \mathrm{de} \\
\end{array}$ & $\begin{array}{l}0.470 \mathrm{c}-\mathrm{e} \\
0.540 \mathrm{bc} \\
\end{array}$ & $\begin{array}{l}0.550 \mathrm{bc} \\
0.590 \mathrm{ab} \\
\end{array}$ & $\begin{array}{c}0.640 \mathrm{a} \\
0.590 \mathrm{ab} \\
\end{array}$ & $\begin{array}{l}0.535 \mathrm{~B} \\
0.543 \mathrm{~B} \\
\end{array}$ \\
\hline \multicolumn{2}{|c|}{ Mean $(A \times C)$} & $\begin{array}{c}0.365 \mathrm{G} \\
0.490 \mathrm{D}-\mathrm{F} \\
0.445 \mathrm{~F} \\
\end{array}$ & $\begin{array}{l}0.460 \mathrm{EF} \\
0.570 \mathrm{BC} \\
0.485 \mathrm{D}-\mathrm{F}\end{array}$ & $\begin{array}{c}0.468 \mathrm{EF} \\
0.600 \mathrm{~B} \\
0.520 \mathrm{C}-\mathrm{E}\end{array}$ & $\begin{array}{c}0.485 \mathrm{D}-\mathrm{F} \\
0.685 \mathrm{~A} \\
0.550 \mathrm{~B}-\mathrm{D}\end{array}$ & & $\begin{array}{l}0.350 \mathrm{C} \\
0.505 \mathrm{~B} \\
0.465 \mathrm{~B} \\
\end{array}$ & $\begin{array}{l}0.480 \mathrm{~B} \\
0.600 \mathrm{~A} \\
0.505 \mathrm{~B} \\
\end{array}$ & $\begin{array}{l}0.488 \mathrm{~B} \\
0.625 \mathrm{~A} \\
0.570 \mathrm{~A} \\
\end{array}$ & \begin{tabular}{|l|}
$0.515 \mathrm{~B}$ \\
$0.600 \mathrm{~A}$ \\
$0.615 \mathrm{~A}$ \\
\end{tabular} & \\
\hline \multicolumn{2}{|c|}{ Mean $(B \times C)$} & $\begin{array}{l}0.470 \mathrm{C} \\
0.397 \mathrm{D} \\
\end{array}$ & $\begin{array}{l}0.507 \mathrm{BC} \\
0.503 \mathrm{BC} \\
\end{array}$ & $\begin{array}{l}0.529 \mathrm{~B} \\
0.530 \mathrm{~B} \\
\end{array}$ & $\begin{array}{l}0.543 \mathrm{~B} \\
0.603 \mathrm{~A} \\
\end{array}$ & & $\begin{array}{l}0.477 \mathrm{C} \\
0.403 \mathrm{D} \\
\end{array}$ & $\begin{array}{l}0.520 \mathrm{~B} \\
0.537 \mathrm{AB} \\
\end{array}$ & $\begin{array}{c}0.553 \mathrm{AB} \\
0.569 \mathrm{~A} \\
\end{array}$ & \begin{tabular}{|l|}
$0.577 \mathrm{~A}$ \\
$0.577 \mathrm{~A}$ \\
\end{tabular} & \\
\hline \multicolumn{2}{|c|}{ Mean ( C ) } & $0.433 C$ & $0.505 \mathrm{~B}$ & $0.529 \mathrm{~B}$ & $0.573 \mathrm{~A}$ & & $0.440 \mathrm{C}$ & $0.528 \mathrm{~B}$ & $0.561 \mathrm{~A}$ & $0.577 \mathrm{~A}$ & \\
\hline \multicolumn{2}{|c|}{ Mean (B) } & \multicolumn{2}{|c|}{$0.512 \mathrm{~A}$} & \multicolumn{2}{|c|}{$0.508 \mathrm{~A}$} & & \multicolumn{2}{|c|}{$0.532 \mathrm{~A}$} & \multicolumn{2}{|c|}{$0.521 \mathrm{~A}$} & \\
\hline & $\operatorname{an} \mathbf{A}$ & $0.445 \mathrm{C}$ & & & $0.500 \mathrm{~B}$ & & $0.458 \mathrm{C}$ & & & $0.539 \mathrm{~B}$ & \\
\hline
\end{tabular}

Mean in each row, Colum or interaction having the same letter (s) are not significantly different at $5 \%$ level.

$A=$ Rootstocks $\quad B=$ Antioxidants $\quad C=$ Concentrations $\quad A \times B=$ Rootstocks $\times$ Antioxidant

$B \times C=$ Antioxidant $\times$ Concentrations $\quad A \times B \times C=$ Rootstocks $\times$ Antioxidant $\times$ Concentrations.

$\mathrm{SA}=$ Salicylic acid $\mathrm{ASC}=$ Ascorbic acid.

$\begin{array}{ll}\text { Element } & \begin{array}{l}\text { Mg } \quad \text { Obreza et al (1992) } \\ \text { (\%) }\end{array} \\ \text { Deficient } & <0.20 \\ \text { Low } & 0.20-0.29 \\ \text { Optimum } & 0.30-0.49 \\ \text { High } & 0.50-0.70 \\ \text { Excess } & >0.70\end{array}$


Table 10. Effect of foliar application of antioxidants and rootstocks on leaf sodium percentage (\%) of Chinese tangerine young trees grown under saline conditions

\begin{tabular}{|c|c|c|c|c|c|c|c|c|c|c|c|}
\hline \multicolumn{2}{|c|}{ Treatments } & \multicolumn{5}{|c|}{ First season, 2015} & \multicolumn{5}{|c|}{ Second season; 2016} \\
\hline Rootstock & Antioxidant & 0 & 400ppm & $600 \mathrm{ppm}$ & $800 p p m$ & MeanAxB & 0 & 400ppm & $600 \mathrm{ppm}$ & $800 p p m$ & MeanAxB \\
\hline \multirow{2}{*}{$\begin{array}{c}\text { Sour } \\
\text { orange }\end{array}$} & SA & $0.110 \mathrm{~g}$ & $0.090 \mathrm{~h}$ & $0.060 \mathrm{i}$ & $0.030 \mathrm{jk}$ & $0.073 \mathrm{D}$ & $0.090 \mathrm{gh}$ & $0.060 \mathrm{i}$ & $0.040 \mathrm{j}$ & $0.020 \mathrm{kl}$ & $0.053 \mathrm{D}$ \\
\hline & ASC & $0.160 \mathrm{e}$ & $0.090 \mathrm{~h}$ & $0.090 \mathrm{~h}$ & $0.040 \mathrm{j}$ & $0.095 \mathrm{C}$ & $0.120 f$ & $0.080 \mathrm{~h}$ & 0.090gh & $0.020 \mathrm{kl}$ & $0.078 \mathrm{C}$ \\
\hline \multirow{2}{*}{$\begin{array}{l}\text { Volkamer } \\
\text { Lemon }\end{array}$} & SA & $0.150 \mathrm{ef}$ & $0.110 \mathrm{~g}$ & $0.040 \mathrm{j}$ & $0.020 \mathrm{kl}$ & $0.080 \mathrm{D}$ & 0.130 ef & $0.100 \mathrm{~g}$ & 0.030jk & $0.020 \mathrm{kl}$ & $0.070 \mathrm{C}$ \\
\hline & ASC & $0.140 f$ & $0.040 \mathrm{j}$ & $0.020 \mathrm{kl}$ & 0.0101 & $0.053 \mathrm{E}$ & $0.077 \mathrm{~h}$ & $0.030 \mathrm{jk}$ & $0.020 \mathrm{kl}$ & 0.0101 & $0.034 \mathrm{E}$ \\
\hline \multirow{2}{*}{ X639 } & SA & $0.180 \mathrm{~d}$ & $0.500 \mathrm{a}$ & $0.300 \mathrm{~b}$ & $0.200 c$ & $0.295 A$ & $0.140 \mathrm{de}$ & $0.400 \mathrm{a}$ & $0.200 c$ & $0.100 \mathrm{~g}$ & $0.210 \mathrm{~B}$ \\
\hline & ASC & $0.160 \mathrm{e}$ & $0.500 a$ & $0.200 \mathrm{c}$ & $0.200 c$ & $0.265 B$ & $0.150 \mathrm{~d}$ & $0.400 \mathrm{a}$ & $0.200 c$ & $0.317 \mathrm{~b}$ & $0.267 \mathrm{~A}$ \\
\hline \multirow{3}{*}{\multicolumn{2}{|c|}{ Mean $(A \times C)$}} & $0.135 \mathrm{E}$ & $0.090 \mathrm{~F}$ & $0.075 \mathrm{G}$ & $0.035 \mathrm{H}$ & & $0.105 \mathrm{D}$ & $0.070 \mathrm{E}$ & $0.065 \mathrm{E}$ & $0.020 \mathrm{~F}$ & \\
\hline & & $0.145 \mathrm{E}$ & $0.075 G$ & $0.030 \mathrm{H}$ & $0.015 I$ & & $0.103 \mathrm{D}$ & $0.065 E$ & $0.025 \mathrm{~F}$ & $0.015 F$ & \\
\hline & & $0.170 \mathrm{D}$ & $0.500 \mathrm{~A}$ & $0.250 \mathrm{~B}$ & $0.200 \mathrm{C}$ & & $0.145 \mathrm{C}$ & $0.400 \mathrm{~A}$ & $0.200 \mathrm{~B}$ & $0.208 \mathrm{~B}$ & \\
\hline \multirow{2}{*}{\multicolumn{2}{|c|}{ Mean $(B \times C)$}} & $0.147 \mathrm{C}$ & $0.233 \mathrm{~A}$ & $0.133 \mathrm{D}$ & $0.083 \mathrm{~F}$ & & $0.120 \mathrm{C}$ & $0.187 \mathrm{~A}$ & $0.090 \mathrm{E}$ & $0.047 \mathrm{~F}$ & \\
\hline & & $0.153 C$ & $0.210 \mathrm{~B}$ & $0.103 \mathrm{E}$ & $0.083 \mathrm{~F}$ & & $0.116 \mathrm{C}$ & $0.170 \mathrm{~B}$ & $0.103 \mathrm{D}$ & $0.116 \mathrm{C}$ & \\
\hline \multicolumn{2}{|c|}{ Mean (C) } & $0.150 \mathrm{~B}$ & $0.222 \mathrm{~A}$ & $0.118 \mathrm{C}$ & $0.083 \mathrm{D}$ & & $0.118 \mathrm{~B}$ & $0.178 \mathrm{~A}$ & $0.097 \mathrm{C}$ & $0.081 \mathrm{D}$ & \\
\hline \multicolumn{2}{|c|}{ Mean (B) } & \multicolumn{2}{|c|}{$0.149 \mathrm{~A}$} & \multicolumn{2}{|c|}{$0.138 \mathrm{~B}$} & & \multicolumn{2}{|c|}{$0.111 \mathrm{~B}$} & \multicolumn{2}{|c|}{$0.126 \mathrm{~A}$} & \\
\hline \multicolumn{2}{|c|}{ Mean A } & $0.084 \mathrm{~B}$ & \multicolumn{2}{|c|}{$0.066 \mathrm{C}$} & $0.280 \mathrm{~A}$ & & $0.065 \mathrm{~B}$ & \multicolumn{2}{|c|}{$0.052 \mathrm{C}$} & $0.238 \mathrm{~A}$ & \\
\hline
\end{tabular}

Mean in each row , colum or interaction having the same letter (s) are not significantly different at $5 \%$ level.

$A=$ Rootstocks $\quad B=$ Antioxidants $\quad C=$ Concentrations $A \times B=$ Rootstocks $\times$ Antioxidant

$\mathrm{B} \times \mathrm{C}=$ Antioxidant $\times$ Concentrations $\quad \mathrm{A} \times \mathrm{B} \times \mathrm{C}=$ Rootstocks $\times$ Antioxidant $\times$ Concentrations

SA= Salicylic acid $A S C=$ Ascorbic acid.

Table 11. Effect of foliar application of antioxidants and rootstocks on leaf chloride percentage $\mathrm{Cl}^{-}(\%)$ of Chinese tangerine young trees grown under saline conditions

\begin{tabular}{|c|c|c|c|c|c|c|c|c|c|c|c|}
\hline \multicolumn{2}{|c|}{ Treatments } & \multicolumn{5}{|c|}{ First season, 2015} & \multicolumn{5}{|c|}{ Second season; 2016} \\
\hline Rootstock & Antioxidant & 0 & 400ppm & $600 \mathrm{ppm}$ & 800ppm & MeanAxB & 0 & 400ppm & $600 \mathrm{ppm}$ & $800 \mathrm{ppm}$ & MeanAxB \\
\hline \multirow{2}{*}{$\begin{array}{l}\text { Sour } \\
\text { orange }\end{array}$} & SA & $0.500 c$ & $0.010 \mathrm{~g}$ & $0.010 \mathrm{~g}$ & $0.010 \mathrm{~g}$ & $0.133 \mathrm{~B}$ & $0.300 c$ & $0.010 f$ & $0.010 f$ & $0.010 f$ & $0.083 \mathrm{C}$ \\
\hline & ASC & $0.400 \mathrm{~d}$ & $0.050 \mathrm{fg}$ & $0.033 \mathrm{fg}$ & $0.010 \mathrm{~g}$ & $0.123 B$ & $0.300 c$ & $0.030 \mathrm{ef}$ & $0.043 \mathrm{ef}$ & $0.010 \mathrm{f}$ & $0.096 \mathrm{C}$ \\
\hline \multirow{2}{*}{$\begin{array}{c}\text { Volkamer } \\
\text { Lemon }\end{array}$} & SA & $0.600 \mathrm{~b}$ & $0.090 \mathrm{fg}$ & $0.040 \mathrm{fg}$ & $0.040 \mathrm{fg}$ & $0.193 \mathrm{~A}$ & $0.200 d$ & $0.080 \mathrm{e}$ & $0.030 \mathrm{ef}$ & $0.020 f$ & $0.0825 \mathrm{C}$ \\
\hline & ASC & $0.600 \mathrm{~b}$ & $0.070 \mathrm{fg}$ & $0.010 \mathrm{~g}$ & $0.010 \mathrm{~g}$ & $0.173 \mathrm{~A}$ & $0.300 \mathrm{c}$ & $0.050 \mathrm{ef}$ & $0.010 f$ & $0.010 \mathrm{f}$ & $0.093 C$ \\
\hline \multirow{2}{*}{ X639 } & SA & $0.700 \mathrm{a}$ & $0.010 \mathrm{~g}$ & $0.010 \mathrm{~g}$ & $0.010 \mathrm{~g}$ & $0.183 \mathrm{~A}$ & $0.500 \mathrm{~b}$ & $0.010 f$ & $0.010 f$ & $0.010 f$ & $0.133 B$ \\
\hline & ASC & $0.100 f$ & $0.200 \mathrm{e}$ & $0.200 \mathrm{e}$ & $0.010 \mathrm{~g}$ & $0.128 \mathrm{~B}$ & $0.600 \mathrm{a}$ & $0.300 \mathrm{c}$ & $0.333 c$ & $0.010 f$ & $0.311 \mathrm{~A}$ \\
\hline \multirow{3}{*}{\multicolumn{2}{|c|}{ Mean $(A \times C)$}} & $0.450 \mathrm{~B}$ & $0.030 \mathrm{DE}$ & $0.022 E$ & $0.010 \mathrm{E}$ & & $0.300 \mathrm{~B}$ & $0.020 \mathrm{~F}$ & $0.027 \mathrm{~F}$ & $0.010 \mathrm{~F}$ & \\
\hline & & $0.600 \mathrm{~A}$ & $0.080 \mathrm{CD}$ & $0.025 \mathrm{E}$ & $0.025 \mathrm{E}$ & & $0.250 \mathrm{C}$ & $0.065 E$ & $0.020 \mathrm{~F}$ & $0.015 \mathrm{~F}$ & \\
\hline & & $0.400 \mathrm{~B}$ & $0.105 \mathrm{C}$ & $0.105 \mathrm{C}$ & $0.010 \mathrm{E}$ & & $0.550 \mathrm{~A}$ & $0.155 \mathrm{D}$ & $0.172 \mathrm{D}$ & $0.010 \mathrm{~F}$ & \\
\hline \multirow{2}{*}{\multicolumn{2}{|c|}{ Mean $(\mathrm{B} \times \mathrm{C})$}} & $0.600 \mathrm{~A}$ & $0.037 \mathrm{D}$ & $0.020 \mathrm{D}$ & $0.020 \mathrm{D}$ & & $0.333 B$ & $0.033 \mathrm{D}$ & $0.017 \mathrm{D}$ & $0.013 \mathrm{D}$ & \\
\hline & & $0.367 \mathrm{~B}$ & $0.107 \mathrm{C}$ & $0.081 \mathrm{C}$ & $0.010 \mathrm{D}$ & & $0.400 \mathrm{~A}$ & $0.127 \mathrm{C}$ & $0.129 \mathrm{C}$ & $0.010 \mathrm{D}$ & \\
\hline \multicolumn{2}{|c|}{ Mean ( C ) } & $0.483 \mathrm{~A}$ & $0.072 B$ & $0.051 \mathrm{~B}$ & $0.015 \mathrm{C}$ & & $0.367 \mathrm{~A}$ & $0.080 \mathrm{~B}$ & $0.073 \mathrm{~B}$ & $0.012 \mathrm{C}$ & \\
\hline \multicolumn{2}{|c|}{ Mean (B) } & \multicolumn{2}{|c|}{$0.169 \mathrm{~A}$} & \multicolumn{2}{|c|}{$0.141 \mathrm{~B}$} & & \multicolumn{2}{|c|}{$0.099 \mathrm{~B}$} & \multicolumn{2}{|c|}{$0.166 \mathrm{~A}$} & \\
\hline \multicolumn{2}{|c|}{ Mean A } & $0.128 \mathrm{C}$ & \multicolumn{2}{|c|}{$0.183 \mathrm{~A}$} & $0.155 \mathrm{~B}$ & & $0.089 \mathrm{~B}$ & \multicolumn{2}{|c|}{$0.088 \mathrm{~A}$} & $0.222 \mathrm{~A}$ & \\
\hline
\end{tabular}

Mean in each row , colum or interaction having the same letter (s) are not significantly different at $5 \%$ level.

$A=$ Rootstocks $\quad B=$ Antioxidants $\quad C=$ Concentrations $A \times B=$ Rootstocks $\times$ Antioxidant

$\mathrm{B} \times \mathrm{C}=$ Antioxidant $\times$ Concentrations $\mathrm{A} \times \mathrm{B} \times \mathrm{C}=$ Rootstocks $\times$ Antioxidant $\times$ Concentrations

$\mathrm{SA}=$ Salicylic acid $\mathrm{ASC}=$ Ascorbic acid.

Arab Univ. J. Agric. Sci., 26(2), 2018 
Effect of foliar application of antioxidants on vegetative growth and leaf mineral content of chinese tangerine young trees budded on some citrus rootstocks grown under saline conditions

accumulation of salt in plant oragns. He also added that under saline condition $\mathrm{Na}$ in flux a cross the plasma lamina to the vacuole might play a major role in permitting turgor maintenance. Some crops showed marked beneficial of sodium $\mathrm{Na}$ specially if the potassium $\mathrm{K}$ supply is limited .Those crops take up large amount of sodium $\mathrm{Na}$ which contributed the osmotic potential of the leaves and increases resistance to water stress salinity. In this respect the effect of salt stress on mineral ions content were recorded by Tsabarducas et al (2015) reported that the highest chloride $\left(\mathrm{Cl}^{-}\right)$and Sodium (Na) quantities have been absorbed by lesson lemon cultivar seedling while the lowest one by Maglini lemon cultivar seedling under salt stress. Kohoshbakht et al (2015) investigated the influence of different salinity concentrations $(0.2,50$ and $75 \mathrm{Mm})$ on nine citrus rootstocks (Cleopatra mandarin, Carrizo citrange, Macrophylla, Iranian mandarin Bakraii, Rangpur lime, Rough lemon, sour orange, swingle citrange and Trifoliate orange) in greenhouse experiment. They found that, ion concentration of $\mathrm{Cl}^{-}$and $\mathrm{Na}$ increased by salinity treatment salinity also increased $\mathrm{Mg}$ content in roots and reduced $\mathrm{Ca}$ and $\mathrm{Mg}$ concentrations in leaves. Also, salinity makes decline in $\mathrm{K}$ concentration. They concluded sour orange and Cleopatra mandarin were the most tolerant rootstocks to salinity of all the nine rootstocks studied. In addition, Trifoliata orange, Carrizo citrange and swingle citrumelo were most sensitive to salt stress followed by the rough lemon and macrophylla.

\subsection{Interaction effect on Mineral ions concen- trations}

Our results show that Table $(5,6,7,8,9$ and 10 ) in both seasons Chinese tangerine budded on volkamer lemon rootstock interacted with antioxidants salicylic acid and/ or ascorbic acid at the concentration 800 ppm and gave the highest nitrogen concentration.

Phosphorus $(\mathrm{P})$, potassium $(\mathrm{K})$, magnesium $(\mathrm{Mg})$ and calcium $(\mathrm{Ca})$ contents affected greatly by the interaction where the highest $\mathrm{Ca}$ Table (8) and Magnesium Table (9) were registered by the interaction between volkamer lemon rootstock interacted with 800 ppm salicylic acid and / or ascorbic acid to give the highest concentration in both seasons. On the other hand the least concentration of $\mathrm{Na}^{+}$was recorded in Chinese tangerine budded on volkamer lemon rootstock interacted with the antioxidants Salicylic acid and /or Ascorbic acid. How- ever the highest $\mathrm{Na}^{+}$achieved by $\mathrm{X} 639$ rootstock interacted with the antioxidants 800 ppm dose.

Phosphorus and Potassium greatly affected by rootstocks, Chinese tangerine budded on sour orange showed the highest concentration of phosphorus (P) Table (6) when interacted with ascorbic acid of the concentration of $600 \mathrm{ppm}$.

Chloride $\mathrm{Cl}$ greatly affected with the interaction between rootstocks, antioxidants and their different concentrations. The highest mean values were recorded for salinized rootstock and zero antioxidants (Control).

We could conclude that salt damage to Chinese tangerine young tress has been mainly attributed to excessive accumulation of chloride $\mathrm{Cl}$ and Sodium $\mathrm{Na}$ in leaves and may be due to nutritional imbalance in plant metabolism.

\section{REFERENCES}

Alhasnawi, A.N.. Ahsan, A.K., Anizan I., Azhar, M., Wan Mohtar, W. and Che, R.I. 2015. Exogenous application of ascorbic acid ameliorates detrimental effects of salt stress in rice (MRQ74 and MR269) seedlings. Asian J. of Crop Sci., 73, 186.

Athar, H.R., Khan, A. and Ashraf, M. 2009. Inducing salt tolerance in wheat by exogenously applied ascorbic acid through different modes. J. Plant Nutr., 32, 1799-1817.

Barth, C., De Tullio, M. and Conklin, P.L. 2006. The role of ascorbic acid in the control of flowering time and the onset of senescence. J. of Experimental Botany, 57(8), 1657-1665.

Brown, J.D. and Lilleland, O. 1946. Rapid determination of potassium and sodium in plant materials and soil extract by flame photometry. Proc. Amer. Soc. Hort. Sci., 48, 341-460.

Çavuşoğlu, K. and Karaferyeli, Ş. 2015. Effects of Ginkgo biloba L. extract on the seed germination, seedling growth and leaf anatomy of barley under saline conditions. Bangladesh J. of Botany, 44(1), 117-123.

Conklin, PI. and Barth, C. 2004. Ascorbic acid a familiar small molecule intertwined in the response of plants to ozone, pathogenies and the onset of senescence. Plant Cell Environ 27, 959-970.

Chapman, H.D. and Pratt, P.E. 1961. Methods of analysis for soil, Plant and water. Univ. Calfornia. Div. of Agric. Sci. pp. 61-62. 
Cottenie, M., Verloo, L., Kiekens, G.V. and Camerlyck, R. 1982. Chemical analysis of plants and soils in laboratory of analytical and Geochemistry. State Univ., Gent. Belgium, pp. 11-33.

Duncan, D.B. 1955. Multiple range and multiple $F$ tests. Biometrics 11, 1-42.

FAO 2016. Statistical Database, www.Fao.org.com /Statistics.

Grieve, A.M., Prior, L.D. and Bevington, K.B. 2007. Long-term effects of saline irrigation water on growth, yield, and fruit quality of 'Valencia orange trees. Crop and Pasture Sci., 58(4), 342-348.

Heikal, M.M.D., Ahmed, A.M. and Shaddad, M.A. 1980. Change in dry weight and mineral composition of some oil producing plants over a range of salinity stresses, Biologia Plantarum, 22(1), 25-33.

Heikal, M.M.D., Ahmed, A.M. and Shaddad, M.A. 1980. Salt tolerance of some oil producing plant. Agric. Belgium 28(3), 437-453.

Hussein, M.M. and Alva, A.K. 2014. Effects of zinc and ascorbic acid application on the growth and photosynthetic pigments of millet plants grown under different salinity. Agricultural Sci., 5(13), 12-53.

Jackson, M.L. 1973. Soil chemical analysis. Prentice-Hall of India Private Limited, New Delhi.

Khoshbakht, D. and Asgharei, M.R. 2015. Influence of foliar-applied salicylic acid on growth, gas-exchange characteristics, and chlorophyll fluorescence in citrus under saline conditions. Photosynthetica, 53(3), 410-418.

Khoshbakht, D., Ramin, A.A. and Baninasab, B. 2015. Effect of sodium chloride stress on gas exchange, chlorophyll content and nutrient concentration of nine citrus rootstocks. Photosynthetica 53(2), 241-249.

Kostopoulou, Z., Therios, I., Roumeliotis, E., Kanellis, A.K. and Molassiotis, A. 2015. Melatonin combined with ascorbic acid provides salt adaptation in Citrus aurantium L. seedlings. Plant Physiology and Biochemistry, 86, 155165.

Muhammed Akbar Anjum. 2008. Effect of Nacl concentration in irrigation water on growth and polyamine metabolism in two citrus rootstocks with different levels of salinity tolerance. Acta Physiol., Plant. 30, 43-52.

Murphy, J. and Riley, J.P. 1962. A modified single solution method of the determination of phosphate in natural water and. Chim. Acta, 27, 13-46.

Noctor, G. and Foyer, C.H. 1998. Ascorbate and glutathione: keeping active oxygen under control. Annual Review of Plant Biology, 49(1), 249-279.

Popova, L., Pancheva, T. and Uzunova, A. 1997. Salicylic acid: properties, biosynthesis and physiological role. Bulg. J. Plant Physiol., 23(1-2), 85-93.

Raskin, I. 1992. Salicylate, a new plant hormone. Plant Physiol. 99, 799-803.

Salma, K.H.A. 2009. Amelioration of $\mathrm{Nacl}$ induced alteration on the plasma membrane of Allium cap L. By ascorbic acid. Aust., J. Basic Appl. Sci., 3, 990-994.

Singh, T.N. and G.H. Snyder (1984). Leaf area index and dry biomass. Taro- Agron., J. 76, 75-76.

Storey, R. 1995. Salt tolerance, ion relation and the effect of root medium on the response of culture to salinity. Aust. Plant Physiol. 22, 101-114.

Storey, R. and Walker, R.R. 1999. Citrus and salinity. Sci., Hort. 78, 39-81.

Syvertsen, J.P., Lloyd, J., McConchie, C., Kriedemann, P.E. and Farquhar, G.D. 1995. On the site of biophysical constraints to $\mathrm{CO}_{2}$ diffusion through the mesophyll of hypostomatous leaves. Plant, Cell and Environment 18, 149-157.

Tsabarducas, V., Chatzisthis, T., Therios, I., Koukourikou-Petridou, M. and Tananaki, C. 2015. Different tolerance of 3 self-rootstock citrus lemon cultivars to NACL stress. Plant Physiology and Biochemistry 97, 196-206.

Watanabe, F.S. and Olsen, S.R. 1965. Test of an ascorbic acid method for determination phosphorus in water and $\mathrm{NaHCo} 3$ extracts form soil, Soil Sci. Soc. Am. Proc., 29, 677-678.

Zikri, M. and Parsonss, L.R. 1992. Salinity tolerance of citrus rootstocks; effect of salt on root and leaf mineral concentration. Plant Soil, 147, 171-181. 